\title{
Lysosomal acid ceramidase ASAH1 controls the transition between invasive and proliferative phenotype in melanoma cells
}

Justine Leclerc ${ }^{1, \#}$, David Garandeau ${ }^{2, \#}$, Charlotte Pandiani ${ }^{1}$, Céline Gaudel ${ }^{1}$, Karine Bille $^{1}$, Nicolas Nottet ${ }^{2}$, Virginie Garcia ${ }^{3}$, Pascal Colosetti ${ }^{4}$, Sophie Pagnotta ${ }^{5}$, Philippe Bahadoran ${ }^{1,6}$, Garance Tondeur $^{7}$, Baharia Mograbi ${ }^{8}$, Stéphane Dalle ${ }^{7}$, Julie Caramel $^{7}$, Thierry Levade ${ }^{3}$, Robert Ballotti ${ }^{1}$, Nathalie Andrieu-Abadie ${ }^{3, \#}$, and Corine Bertolotto ${ }^{1, \#, *}$

1, Université Côte d'Azur, Inserm U1065, C3M, Nice, France, Team 1, Biology and pathologies of melanocytes. Equipe labellisée ARC 2015

2, Université Côte d'Azur, Inserm U1065, C3M, Nice, France

3, Université Toulouse III, Toulouse, Inserm, UMR1037, CRCT, Toulouse, F-31037, France, Team 4, Sphingolipids, metabolism, cell death and tumor progression

4, CarMeN Laboratory, INSERM U1060, INRA1397, INSA, Lyon, France

5, Université Côte d'Azur, Centre Commun de Microscopie Appliquée, Nice, France

6, CHU NICE, Département de Dermatologie, Nice, France

7, Université de Lyon, Inserm, U1052, CNRS 5286, Equipe Labellisée Ligue contre le Cancer, Lyon, France.

8, INSERM, ERI21/EA 4319, Nice, F-06107, France

\# the authors have equally contributed to the work

*Correspondence should be addressed to Corine Bertolotto: bertolot@unice.fr

Running title: ASAH1 is a driver of the phenotypic switch in melanoma 


\section{Abstract}

Phenotypic plasticity and subsequent generation of intratumoral heterogeneity underly key traits in malignant melanoma such as drug resistance and metastasis.

Melanoma plasticity promotes a switch between proliferative and invasive phenotypes characterized by different transcriptional programs of which MITF is a critical regulator.

Here, we show that the acid ceramidase ASAH1, which controls sphingolipid metabolism acted as a rheostat of the phenotypic switch in melanoma cells. Low ASAH1 expression was associated with an invasive behavior mediated by activation of the integrin alphavbeta5-FAK signaling cascade. In line with that, human melanoma biopsies revealed heterogeneous staining of ASAH1 and low ASAH1 expression at the melanoma invasive front. We also identified ASAH1 as a new target of MITF, thereby involving MITF in the regulation of sphingolipid metabolism. Together, our findings provide new cues to the mechanisms underlying the phenotypic plasticity of melanoma cells and identify new anti-metastatic targets.

Key words: melanoma, plasticity, MITF, ASAH1 


\section{Introduction}

Cancer cells including melanoma cells display a high degree of intertumoral heterogeneity as well as intratumoral heterogeneity (ITH) ${ }^{1,2}$. ITH is dynamically controlled by the tumor microenvironment, but also by the intrinsic plastic capacity of the cells and is recognized as the main therapy resistance mechanism. Therefore, the molecular mechanisms behind the plastic capacity of cancer cells and the underlying heterogeneity must be fully elucidated in order to develop effective and lasting therapies.

Melanoma cells can be classified into at least two major states, i.e proliferative and invasive. A model derived from these findings, the "phenotype-switching" model, predicts that melanoma cells may switch between these two states ${ }^{3}$. ITH can be, at least in part, understood by this phenotypic switch. Most importantly, melanoma phenotype switching is associated with intrinsic and acquired resistance to targeted therapies, which is the major cause for the resultant mortality of patients with cancer 4-7. It also negatively impacts on immune checkpoint blockade and impairs immunotherapy efficiency ${ }^{8,9}$. The lineage specific transcription factor MITF, which acts as a bona fide melanoma oncogene ${ }^{10-13}$, is a key driver of the transition between the proliferative and the invasive state of melanoma cells ${ }^{14-17}$. However, how MITF regulates the phenotypic switch of melanoma cells is not fully elucidated.

Cancer cells rewire their metabolic pathways to satisfy their abnormal demands for proliferation and survival ${ }^{18}$. In cancer cells, the most common metabolic change is the use of glycolysis even in normoxic condition, a phenomenon called "Warburg effect" ${ }^{19}$. There are a number of targets involved in glycolysis, which are currently exploited for potential therapeutic drug strategies ${ }^{20}$. However, as functionally plastic 
cells, malignant melanoma cells can rewire their metabolism towards oxidative phosphorylation, or glutaminolysis, two processes limiting drug efficacy $8,21,22$. Several lines of evidence indicate that cancer cells also show alterations in other metabolic pathways such as lipid metabolism ${ }^{23,24}$. In melanoma, the role of lipid metabolism is poorly elucidated.

Here, by comparing the transcriptomic profile of invasive and proliferative melanoma cells, we found that the expression of acid ceramidase ASAH1, a key enzyme of sphingolipid metabolism ${ }^{25}$, was among the top differentially expressed genes, suggesting that ASAH1 might be critical to the phenotypic switch. We performed a comprehensive characterization of ASAH1, function and regulation in melanoma. We found that ASAH1 loss drove the transcriptional reprogramming of proliferative melanoma cells into an invasive cell subpopulation characterized by a reduced expression of E-cadherin and activation of the integrin alphaV/beta5-FAK signaling cascade. Conversely, forced expression of ASAH1 in invasive melanoma cells reduced their motile features and rendered them more proliferative. We also identified ASAH1 as a target of MITF, which is a key driver in the phenotypic transition. These data also causally involve for the first time MITF in the regulation of sphingolipid metabolism.

Together, our findings reveal that lysosomal ASAH1 and the sphingolipid metabolism drive the transition between the proliferative and the invasive phenotype in melanoma cells, which is at the origin of intratumoral heterogeneity and a source of therapy resistance. 


\section{Results}

\section{ASAH1 controls the phenotype switching of melanoma cells.}

To identify genes differentially expressed between proliferative and invasive melanoma cells, we performed transcriptomic analysis. Using both melanoma cell lines and melanoma cells freshly isolated from human biopsies we found that the acid ceramidase ASAH1, which is located at the lysosome and which controls the sphingolipid metabolism, was among the genes most differentially expressed between the two phenotypes (Figure 1A). We confirmed this observation using data from another source (Supplemental figure 1A) ${ }^{26}$. Further, analysis of publicly available datasets revealed significantly higher expression of ASAH1 in melanoma cells compared to other human cancer cell lines, strengthening the key role of ASAH1 in melanoma cells (Figure 1B).

Analysis of publicly available datasets showed a positive correlation between ASAH1 and the transcription factor MITF (Figure 1C and Supplemental figure 1B).

ASAH1 expression was next studied by QPCR in another set of melanoma cell lines expressing MITF at different levels. Two groups of melanoma cells were distinguished, one expressing low to very low mRNA level of ASAH1 (ASAH1 ${ }^{\text {low }}$ ) and one expressing high mRNA level of ASAH1 (ASAH1 ${ }^{\text {high }}$ ) (Supplemental figure $1 C$ ). We next assessed ASAH1 at the protein level. ASAH1 is a glycoprotein processed from a 55-kDa precursor into an active heterodimeric enzyme with alpha $(\alpha, 14-\mathrm{kDa})$ and beta $(\beta, 37-k D a)$ subunits ${ }^{27}$. Immunoblots with a homemade antibody ${ }^{28}$ easily detected the proform and the $\alpha$ and $\beta$ active subunits (lanes 4-6 and lanes 10-12) in melanoma cells with high ASAH1 mRNA expression, whereas the different forms were hardly detectable in cells with low ASAH1 mRNA (lanes 7-9) (Figure 1D). 
Because of homemade antibody restriction, we also tested two commercially available antibodies. Only one antibody gave positive results leading to the detection of the proform and of the $\beta$-subunit (Supplemental figure 1D). The different melanoma cell lines analysed for MITF expression resulted in the detection of two groups with low/absent MITF expression (MITF ${ }^{\text {low }}$ ) and high MITF expression $\left(\mathrm{MITF}^{\text {high }}\right)$ (Figures $1 \mathrm{E}$ and supplemental figure 1E).

Using the fluorogenic substrate Rbm14-12 ${ }^{29}$, we measured the activity of ASAH1, which varied accordingly with the level of expression (Figure 1F). We next performed a comprehensive lipidomic analysis, using ultra-performance liquid chromatography/mass spectrometry. Lipidomic analysis revealed higher sphingosine level in $\mathrm{ASAH} 1^{\text {high }}$ cells, consistent with an ASAH1 activity, compared to ASAH1 low cells (Figure 1G). Of note, we found no correlation between expression of ASAH1 and the BRAF mutational status.

Together, our results indicate that ASAH1 is highly expressed and active in about $60 \%$ of melanoma cells and its expression correlates with that of MITF.

\section{ASAH1 expression in human melanoma samples}

Immunohistochemical staining of MITF and ASAH1 proteins were performed in a series of human melanoma samples, including primary malignant melanoma, and metastatic samples. Areas of high and low MITF and ASAH1 expression were observed in cutaneous metastasis of melanoma (Figures $2 \mathrm{~A}-\mathrm{B}$ ). Thus, the correlation between MITF and ASAH1 expression was also observed in vivo.

Interestingly, within thick primary melanomas, a distinct gradient was observed, where nuclear MITF and cytoplasmic ASAH1 were strongly expressed in superficial sites with weaker or absent staining at the invasive sites of the lesion (Figure $2 \mathrm{C}$ ), 
strengthening the notion that ASAH1 loss correlates with a loss of MITF and a gain of motile ability and aggressiveness. In conclusion, our results confirm in vivo the correlation between MITF and ASAH1.

\section{MITF drives expression of ASAH1}

Our previous observations suggested that ASAH1 was a downstream target of MITF. To assess this hypothesis, we silenced MITF with two different siRNA. As expected, the siRNA reduced the expression of mRNA for MITF and its target genes MLANA and TYR. MITF inhibition also strongly decreased ASAH1 mRNA (Figure 3A and supplemental figures $2 A-B)$. Similar observations were obtained the protein level showing that MITF inhibition caused a reduction in the proform and the $\beta$-active subunit of ASAH1 (Figure 3B). Accordingly, MITF inhibition reduced ASAH1 activity (Figure 3C) and consequently the quantity of sphingosine (SPH) (Supplemental figure $2 \mathrm{C})$.

Conversely, we assessed whether MITF was sufficient to drive the expression of ASAH1. Forced expression of MITF in two different melanocyte cells, as shown by immunoblot (Figure 3D and supplemental figure 2D), enhanced the expression and activity of ASAH1 (Figure 3E and supplemental figure 2E) compared with cells transduced with an empty vector (Figure $3 F$ ). MITF is a transcription factor that binds to DNA to control the expression of its targets. Using recently described MITF chromatin immunoprecipitation sequencing (ChIP-seq) data set ${ }^{30}$, we identified significantly enriched MITF-binding sites in the genomic region of $A S A H 1$ (Figure $3 G)$. MITF-binding at the promoter region overlapped with the binding of H3K27ac and $\mathrm{H} 3 \mathrm{~K} 4 \mathrm{me} 3$, two histone marks associated with active transcription ${ }^{31}$, thereby indicating that MITF controlled the expression of ASAH1 at the transcriptional level. 
In line with that, MITF stimulated the activity of $A S A H 1$ promoter (Supplemental figure $2 F$ ).

We next addressed the functional importance of ASAH1 in MITF effects. Forced expression of ASAH1 rescued the reduction of cell proliferation mediated by MITF inhibition. Forced expression of ASAH1 also slightly enhanced the number of cells compared to the control condition (Figure 3Ha). Further, an increase expression in the cell cycle inhibitor p27 was observed upon MITF inhibition by siRNA, but did not occur in these cells when they were transduced with ASAH1. Together, these results demonstrate the causal role of ASAH1 in MITF proliferative activity (Figure $3 \mathrm{Hb}$ ). Almost no cell death was found in these conditions (Supplemental figure $2 \mathrm{G}$ ). Of note, immunoblot also showed that forced expression of ASAH1 was associated with an increase in MITF expression, thereby suggesting a bidirectional regulation between MITF and ASAH1.

Collectively, our findings identify ASAH1 as a new MITF target gene and demonstrate for the first time the involvement of MITF in the control of sphingolipid metabolism.

ASAH1 controls the switch between the proliferative and invasive phenotype in melanoma cells.

We next assessed the impact of ASAH1 knockdown in $\mathrm{ASAH} 1^{\text {high }}$ cells on short-term cell growth, colony formation assay and apoptosis detection in various melanoma lines transfected with siRNA against ASAH1 versus a control siRNA. To rule out nonspecific effects, different siRNA were used. As expected, the siRNA triggered a strong decrease in expression of mRNA and protein for ASAH1 (Figure 4A and Supplemental figure $3 \mathrm{~A}$ ) and translated into a decrease in ASAH1 activity 
(Supplemental Figure 3B). Of note, siASAH1\#2 displayed stronger effect than siASAH1\#1 and was used in the following experiments as siASAH1. Electron microscopy revealed that ASAH1-inhibited melanoma cells compared to control cells were characterized by an accumulation of intracellular vacuoles rich in sphingolipid (Supplemental figure 3C) typically resembling the phenotype of cells from patients with lysosomal storage disorders ${ }^{32}$. ASAH1-inhibition dramatically reduced the number of cells in short-term cell growth (Supplemental figure 3D) or in colony formation assays (Supplemental figure 3E). Remarkably, the growth arrest caused by ASAH1 reduction was comparable to that of MITF inhibition, in agreement with ASAH1 being a target gene of MITF (Supplemental figures 3D-E). FACS analysis of DAPI stained cells did not reveal a significant difference in cell death between control cells or cells in which ASAH1 or MITF-were inhibited (Supplemental figure 3F).

Conversely, we investigated the effect of ASAH1 forced expression in ASAH1 1ow cells. ASAH1 up-regulation in WM3918 cells was confirmed by Q-PCR and immunoblot (Supplemental figures 4A-B). Further, forced expression of ASAH1 did not change its cellular localization (Supplemental figure $4 \mathrm{C}$ ) and it translated into more ASAH1 activity (Supplemental figure 4D). Melanoma cells with forced expression of ASAH1 proliferated faster than native parental control cells (Supplemental figure 4E) and displayed a reduced motility (Supplemental figure 4F). Analysis of the TCGA-SKCM dataset, revealed accordingly, an association of ASAH1 with the proliferative signature described in Verfaillie et al. ${ }^{33}$ (Supplemental figure $4 G)$.

In addition, ASAH1-inhibited cells underwent morphological changes. They exhibited an increased dendritic morphology (Supplemental figure 5A). Moreover, adherence monitored either by microscopic observation after seeding (Supplemental figures 5B- 
C) or monitored in real-time using the xCELLigence technology showed that cells with reduced ASAH1 expression adhered faster than that with high ASAH1 expression (Supplemental figure 5D). Similar observations were obtained in cells transfected with MITF siRNA (Supplemental figure 5D). These changes suggested that ASAH1 controled the motile ability of melanoma cells.

Boyden chamber motility studies and scratch wound assays in different melanoma cell lines demonstrated that ASAH1 reduction enhanced cell migration compared to control cells (Figure 4B and supplemental figures 5E-F). ASAH1-reduction stimulated cell migration to the same extent as MITF inhibition. Conversely, melanoma cells with forced expression of ASAH1 displayed reduced motile ability (Supplemental figure 4F). In line with that, MITF and ASAH1 low melanoma cells have higher invasive capacities than MITF and ASAH1 high cells (Supplemental Figure 5G). To demonstrate that the observed effects were dependent on ASAH1 activity, exogenous sphingosine was used to restore functional ASAH1 metabolic cascade. Short-term exposure to sphingosine at concentrations from 1-5 $\mu \mathrm{M}$ had no effect on cell number (Supplemental figure 6A-B). However, it dramatically reduced melanoma cell migration induced by the loss of ASAH1 or MITF (Figure 4C and supplemental figures $6 \mathrm{C}-\mathrm{D}$ ) and impaired migration of $\mathrm{ASAH} 1^{1 \mathrm{low}}$ melanoma cells (Figure 4D).

Epithelial-to-mesenchymal transition (EMT)-inducing transcription factors enhance mesenchymal (loss of epithelial characteristics such as E-cadherin expression) and motile features. TWIST1 is an EMT-controlling factor particularly important in melanoma ${ }^{34}$. Interestingly, we observed that ASAH1-reduction in ASAH1 ${ }^{\text {high }}$ cells enhanced TWIST1 expression (Supplemental figure 7A) and caused a loss of Ecadherin expression (Supplemental figure 7B), whereas ASAH1 forced expression in ASAH1 ${ }^{\text {low }}$ cells had the opposite effect, reducing TWIST1 (Supplemental figure 7C) 
and stimulating E-cadherin expression (Supplemental figure 7D). These features translated into an enhanced sphere formation ability of ASAH1-inhibited melanoma cells (Supplemental Figure 7E). Thus, ASAH1 impacts on the mechanical and mesenchymal features of melanoma cells. Collectively, our findings demonstrate that ASAH1 acts as a rheostat to control the switch between proliferative and invasive states in melanoma cells.

\section{ASAH1, through activation of the ITG $\alpha \mathrm{V} \beta 5 / \mathrm{FAK}$ signaling cascade, controls motility of melanoma cells.}

To broadly dissect the molecular mechanisms by which ASAH1 exerts its effect in melanoma cells, we performed a gene expression profiling of ASAH1-knocked down cells compared to control cells. Top canonical pathways of the differentially expressed genes captured in David tool were related to cell migration and trafficking such as "pathways in cancer", "focal adhesion", and "ECM-receptor interaction" (Figure 5A), thereby strengthening the observation that ASAH1 loss is associated with an enhanced motile capacity of melanoma cells. Of note, our transcriptomic analysis also revealed a reduction in expression of several MITF target genes. The expression in MITF mRNA itself was slightly downregulated in the three cell lines but did not reach the statistical threshold. Nevertheless, at the protein level, forced expression of ASAH1 enhanced MITF (Figure $3 \mathrm{Hb}$ ) and ASAH1 siRNA reduced MITF expression (Supplemental Figure 7F).

We next focused our attention on the focal adhesion kinase FAK, which is linked to integrin signals to promote cell migration. Immunoblot showed that ASAH1 silencing in different cell types resulted in a significant increase in FAK phosphorylation (Figure 5B). Such treatment had no effect on the levels of FAK protein. Immunofluorescence 
staining of cells following ASAH1 siRNA treatment revealed that FAK localized to focal adhesions compared to control cells in which FAK was more cytoplasmic (Figure 5C and supplemental figure 8A). Conversely, forced expression of ASAH1 inhibited FAK phosphorylation (Figure 5D). Gene expression profiling revealed the increased expression of integrin beta 5 that was confirmed at the protein level (ITG $\beta 5$ ) (Figure 5E). ITG $\beta 5$ can associate with integrin alpha $V$ (ITG $\alpha \mathrm{V}$ ). Transcriptomic profiling showed a small increase in expression of mRNA for ITGAV in ASAH1-inhibited cells, even though the difference did not reach statistical significance. However, a statistically significant difference in ITGAV mRNA between control and ASAH1-inhibited cells was observed by QPCR and was strenghtened at the protein level by western blot (Figure 5E and supplemental figure 8B). Further, both integrins play critical role in cell migration, invasion and tumorigenesis. Q-PCR and immunoblot revealed the recurrent increased expression in ITG $\alpha$ V upon ASAH1 inhibition in different melanoma cells (Figure 5E). As shown by immunofluorescence, ITG 35 staining was confined predominantly to the cellular periphery in ASAH1knocked down cells compared to its cytoplasmic localization in control cells (Figure $5 F)$. In cells treated with MITF siRNA, ITG $\beta 5$ generated a pattern of staining similar to that obtained with ASAH1-inhibition (Figure 5F). We next thought to determine whether ITG $\alpha \mathrm{V} \beta 5$ was causally involved in melanoma cell migration mediated by ASAH1 inhibition. Whereas treatment with an ITG $\alpha \mathrm{V} \beta 5$ blocking antibody had no effect on cell survival (Supplemental figure $8 \mathrm{C}$ ), it dramatically impaired melanoma cell migration (Figure $5 \mathrm{G}$ and supplemental figures 8D-E). ITG $\alpha \mathrm{V} \beta 5$ blocking antibody exhibited similar causal effect in melanoma cell migration mediated by MITF inhibition. Altogether, our data demonstrate that ASAH1 controls melanoma cell plasticity and migration through integrin-mediated FAK activation. 


\section{Discussion}

Here, we demonstrate that the lysosomal acid ceramidase ASAH1, which controls sphingolipid metabolism, acts as a driver of the phenotypic switch, thereby contributing to an intratumoral heterogeneity. We showed that whereas melanoma cells with high expression of ASAH1 displayed a proliferative activity, low ASAH1 cells had enhanced motile ability. ASAH1 was previously shown to play an important role in melanoma proliferation ${ }^{35}$. We also identified ASAH1 as a target of MITF in melanoma cells, thereby implying for the first time MITF in the regulation of sphingolipid metabolism. Until now, an enhanced transcription of ASAH1 has been reported by cAMP-responsive element binding protein (CREB) in human adrenal corticocarcinoma cells ${ }^{36}$ and by ERa and Sp1 in breast cancer cells ${ }^{37}$. Thus, our study adds new clues to the regulation of ASAH1.

Elevated ASAH1 expression has been reported in various types of human cancers such as prostate, breast, and head and neck ${ }^{38,39}$ and has been associated to increased malignancy and worse clinical outcome ${ }^{40-42}$. However, ASAH1 expression correlates with a good prognosis in ER-positive breast cancer ${ }^{43}, 44$, thereby indicating that high ASAH1 expression is not necessarily an indicator of unfavorable tumor features and lower survival.

Further, defects in the ASAH1 gene result in the lysosomal storage disease Farber (FD) lipogranulomatosis, with an invariably fatal outcome ${ }^{45}$. FD patients are not prone to develop cancer. However, individual with the most common, "classical" form of FD die at approximately 2 years of age, before melanoma can develop. The data gathered here on the role of ASAH1 in melanoma cells highlighted genes and signaling cascades that might serve as new potential therapeutic targets in the FD patients. 
The pro- or anti-tumoral role of ASAH1 can be explained by tissue-specific functions or by its involvement during specific stages of cancer. Supporting this idea, MITF expression must transiently decrease to enable melanoma cell migration and invasion but high MITF expression must be recovered to allow the growth of the metastases ${ }^{14,16,46}$. In response to microenvironmental signals, MITF exhibits an oscillatory pattern of expression and melanoma cells switch back-and-forth between proliferative and invasive to drive the metastatic disease ${ }^{3,8,16}$. ASAH1 might follow the same oscillatory model as MITF.

MITF-silenced melanoma cells also display features of senescence ${ }^{30,47}$. ASAH1 depleted cells were positive for the senescence associated $\beta$-galactosidase staining (Supplemental figure $7 G$ ), in agreement with a previous report ${ }^{48}$, and also displayed traces of the pro-inflammatory secretome, illustrated by an increase expression of CCL2 and CYR61, as we previously reported in MITF low cells ${ }^{49,50}$. Melanoma cells with reduced expression of ASAH1 exhibited a molecular program linked to invasion that partially overlapped that of MITF loss. Transcriptomic analysis of melanoma cells transfected with ASAH1-siRNA revealed no change compared to control cells in ZEB1 or SNAIL and an increase in TWIST1, BRN2 or SLUG expression, which are critical factors for melanoma progression and metastasis ${ }^{34,51,52}$. Additionally, only IGF1R out of other critical tyrosine kinase receptors (PDGFRB, AXL, EGFR, ERBB3, EPHA2), that were reported to be deregulated in the MITF low invasive phenotype ${ }^{6}$, 8, 53, was enhanced in ASAH1-silenced melanoma cells. Thus, the gene expression signature of ASAH1-inhibited cells appeared intermediate between the proliferative and invasive phenotypes reported for MITF. This is consistent with previous reports showing that a subgroup of profiled melanoma cell lines was intermediate between the two states ${ }^{26,54}$. Further, melanoma cells with both proliferative and invasive 
phenotypes have been recently described ${ }^{55}$.

Noteworthy, melanoma cells expressing classical markers of senescence were reported to retain their ability to migrate and invade ${ }^{56}$. This is consistent with our findings demonstrating that ASAH1 loss induced a senescence-like phenotype and enhanced the motile ability of melanoma cells. Lai et al. also showed that ASAH1-null cells lose the ability to form cancer-initiating cells. Contrastingly, we demonstrated that cells in which ASAH1 expression was inhibited via siRNA gain a motile mesenchymal phenotype, which is a feature of cancer stem populations. This discrepancy can be explained by the complete compared to transient inhibition of ASAH1 that prevents adaptation through plastic ability of melanoma cells. The partial loss-of-function phenotype generated by RNAi may more accurately recapitulate the effects of microenvironment than the complete loss-of-function phenotype. Furthermore, the transcriptomic analysis revealed that ASAH1 inhibition by siRNA triggered a slight decrease in expression of mRNA for MITF, yet it did not reach the statistical threshold. Nevertheless, at the protein level, forced expression of ASAH1 enhanced MITF expression and ASAH1 siRNA reduced MITF expression. These observations suggested a complex post-transcriptional regulation of MITF by ASAH1 that needs further studies to be elucidated. Migration requires the coordinated regulation of both E-cadherin-mediated cell-cell adhesions and integrin-mediated adhesions that contact the surrounding extracellular matrix $(\mathrm{ECM})^{57}$. In line with that, E-cadherin inhibition and increased expression of TWIST1, a known E-cadherin transcriptional repressor ${ }^{34,58}$, paralleled ASAH1 loss. Supporting our data, sphingosine-1-phosphate (S1P) production, which is produced by ASAH1 activity, has been reported to suppress migration of breast cancer cells through E-cadherin re-expression and enhanced cell-cell adhesion ${ }^{59}$. Besides, we observed that ASAH1 
loss favored anchorage-independent growth, correlates with acquisition of mesenchymal properties and with integrin activation. Accordingly, cells with reduced ASAH1 expression displayed heightened integrin and FAK signaling. Among integrin, we identified integrin $\alpha \mathrm{V} \beta 5$ as the main dimer responsible for melanoma cell migration mediated by ASAH1-inhibition. In highly invasive melanoma cells, integrin $\alpha \mathrm{V} \beta 5$ was also shown to regulate VEGF-A secretion and activation of the vascular endothelium, a key step in the extravasation process and formation of distant metastasis ${ }^{60}$. Our transcriptomic analysis revealed that VEGF-C is one of the top deregulated genes upon ASAH1 reduction. Additional candidates that may reflect aspects of migration and heterogeneity of melanoma cells include several transcription factors, such as BRN2 and NFIB, that were found increased in our transcriptomic analysis of ASAH1-inhibited cells. Recently, NFIB has been shown to mediate BRN2 driven melanoma cell migration and invasion through upregulation of EZH2 and downregulation of MITF ${ }^{61,62}$. However, in our trancriptomic analysis EZH2 mRNA expression was inhibited after ASAH1 inhibition by siRNA, instead of being increased as reported in response to BRN2 forced expression ${ }^{61}$. Thus, these observations suggest that the increase in motile and invasive properties evoked by ASAH1 inhibition and by BRN2 forced expression are different.

Altogether, we show that ASAH1 influences the phenotypic switch in melanoma cells and that melanoma cells acquire motile properties after the loss of ASAH1. Further, our findings point out to a link between metabolism of sphingolipid and melanoma cell plasticity and open new therapeutic perspectives in melanoma. 


\section{Experimental procedures}

\section{Cell cultures and reagent}

All human melanoma cells were cultured in DMEM supplemented with $7 \%$ FCS at $37^{\circ} \mathrm{C}$ in a humidified $\mathrm{CO} 2$ incubator. The human melanoma cell line WM3912, WM8 and WM3918 are a gift of Dr. M. Herlyn (The Wistar Institute, Philadelphia, PA). Other melanoma cell lines were purchased from the American Tissue Culture Collection. All cell lines were regularly tested for mycoplasma contamination.

\section{Human tumor samples and immunohistochemical analyses}

Twenty $\mathrm{BRAF}^{\mathrm{V} 600}$-mutated melanoma tumor samples were obtained through the Biological Resource Center of the Hôpital Lyon Sud, Hospices Civils de Lyon and were used with the patient's written informed consent. This study was approved by the scientific board of the Hospices Civils de Lyon. Formalin-fixed, paraffinembedded and $3 \mu$ m-thick tissue sections underwent immunohistochemical (IHC) staining using commercially available antibodies against MITF (C5/D5, mouse monoclonal, 1/200, Roche) and ASAH1 (ab74469, 1/100, Abcam, Cambridge, MA, USA) and Ultraview red detection or DAB revelation. For MITF, the staining was nuclear while for ASAH1 the staining was cytoplasmic. A blinded evaluation of the staining was carried out by experienced pathologists.

\section{Gain and loss of function experiments}

Briefly, cells were transfected with $20 \mathrm{nM}$ of siRNA plus $5 \mu$ lipofectamine ${ }^{\mathrm{TM}}$ RNAiMAX in opti-MEM medium (Invitrogen, San Diego, CA, USA). Control (siC) and siRNAs for MITF were previously described ${ }^{63}$. siRNA to ASAH1 were from Invitrogen. 
Cells were infected with lentiviral particules for ASAH1 and when indicated $24 \mathrm{hrs}$ later they were transfected with control or MITF siRNA for $72 \mathrm{hrs}$.

\section{Cell count and viability}

Cell count and viability were determined in flow cytometry by adding DAPI at $1 \mu \mathrm{g} / \mathrm{ml}$ and for a short time to the population of cells.

\section{Colony formation assay}

501mel melanoma cells were transfected with control or ASAH1 siRNA for 48hrs. Cells were trypsinized, seeded in 6 -well plates $\left(3 \times 10^{3}\right)$ and were then placed in a $37^{\circ} \mathrm{C}, 5 \% \mathrm{CO}_{2}$ incubator. Colonies of cells were stained with $0.04 \%$ crystal violet $/ 2 \%$ ethanol in PBS for 30 min after for 14 days. Picture of crystal violet stained wells were taken. The colony formation assay was performed in triplicate.

\section{Anchorage-independent growth}

501mel melanoma cells were transfected with control or siRNA to ASAH1 and next subjected to a 2-layer soft agar assay in 6 -well plates. Cells $\left(1.5 \times 10^{4}\right)$ were mixed with $2 \mathrm{ml}$ of $1 \mathrm{X}$ culture medium with $10 \%$ FBS and $0.3 \%$ agar, cooled to $42^{\circ} \mathrm{C}$, and plated on top of a solidified bottom layer of $2 \mathrm{ml}$ of $1 \%$ noble agar in cell respective $1 \mathrm{X}$ culture medium. Every 2 days, $1 \mathrm{ml}$ of growth medium was added into the wells. One week later, bright-field images were taken using a Zeiss Axiovert S100. The number of colonies grown in soft agar was quantified, and the colony size (i.e., image area) was calculated using Zeiss ZEN software.

\section{LC/MS Analysis of sphingolipids and ASAH1 activity}


Sphingolipid were extracted and prepared as published ${ }^{64}$ and analyzed by LC/MS as previously described ${ }^{29}$. ASAH1 activity was measured as previously reported ${ }^{29}$

\section{Immunofluorescence and confocal experiments}

Cells were fixed and permeabilized as previously described ${ }^{49}$ before being exposed to anti-ASAH1 1/100 (Sigma, HPA005468) antibodies and then with 1:1000 dilution anti-rabbit Alexa Fluor 594 labeled secondary antibody (Invitrogen, San Diego, CA, USA). Confocal optical sections of cells were obtained with a Zeiss LSM510Meta microscope (Zeiss, Göttingen Germany) using a X63 NA1.4 Plan-Apochromat oil immersion lens. The parameters of the system were adjusted to avoid saturation.

\section{Western blot assays}

Western blots were carried out as previously described ${ }^{46}$. Cell lysates $(30 \mu \mathrm{g})$ were separated by SDS-PAGE, transferred onto a PVDF membrane and then probed with antibodies to MITF (Abcam, Ab12039), ASAH1 (Sigma, HPA005468), ERK2 (Santa Cruz biotechnology, clone D-2), phospho-ERK1/2 (Thr202/Tyr204) (Cell Signaling Technology Inc., \#2370), anti-phosphoFAK (Cell Signaling Technology Inc., \#3283), total FAK (Millipore, clone 4.47), p27 (Ozyme, \#3686), ITG $\beta 5$ (Cell Signaling Technology Inc., D24A5), ITG $\alpha$ V (Santa Cruz biotechnology, p2W7), to integrin alpha V+beta 5 (Abcam, ab24694), E-cadherin (Ozyme, \#5296) and HSP90 (Santa Cruz biotechnology, sc-13119). Horseradish peroxidase-conjugated anti-rabbit or antimouse antibodies were from Dakopatts (Glostrup, Denmark). Proteins were visualized with the ECL system (Amersham). The western blots shown are representative of at least 3 independent experiments. 


\section{Migration Assays}

Cell migration was carried out using a Boyden chamber assay with 8-um pore filter inserts (BD Bioscience). Cells were seeded on the upper chamber of a trans-well and DMEM 7\%FBS placed into the lower chamber. Cells adherent to the underside of the filters were fixed with $4 \%$ PFA, stained with $0.4 \%$ crystal violet and five random fields at x20 magnification were counted. Results represent the average of triplicate samples from three independent experiments.

Cell migration was also studied in classical wound healing assays. Cells were grown to form a confluent monolayer and next were wounded with a p200 pipette tip. Pictures (10X) of the same areas were recorded using an Axiovert $200 \mathrm{M}$ microscope (Zeiss) equipped with a CoolSnap ${ }^{\mathrm{TM}}$ ES camera (Photometric ${ }^{\circledR}$, Roper Scientific).

\section{mRNA preparation, Real-time/quantitative PCR}

mRNA isolation was carried out with Trizol (Invitrogen), according to standard procedure. QRT-PCR was carried out with SYBR® Green I and Multiscribe Reverse Transcriptase (Applied Biosystems) and monitored by an ABI Prism 7900 Sequence Detection System (Applied Biosystems, Foster City, CA). Detection of SB34 gene was used to normalize the results. Primers were designed for each cDNA with Primer Express Software (Applied Biosystems) and are available upon request.

\section{Gene expression profiling and bioinformatics}

Total RNA of three different cell lines transfected with control scrambled siRNA or siRNA to ASAH1 was isolated with TRIZOL. Integrity of RNA was assessed by using an Agilent BioAnalyser 2100 (Agilent Technologies) (RIN above 8). RNA samples were then labeled with Cy3 dye using the low RNA input QuickAmp kit (Agilent) as 
recommended by the supplier. $600 \mathrm{ng}$ of labeled cRNA probe were hybridized on $8 \times 60 \mathrm{~K}$ high density SurePrint G3 gene expression human Agilent microarrays. Data analyses were performed using R (http://www.r-project.org/). The quality control was performed using the Bioconductor package arrayQualityMetrics and custom $\mathrm{R}$ scripts. We used a linear modeling approach to calculate log ratios, moderated $t$ statistics, log odds ratios of differential expression (B statistic), and $p$ values for each comparison of interest. $P$ values were adjusted for multiple testing using the Benjamini and Hochberg method, which controls the false discovery rate. Differentially expressed genes were selected based on AbsLogFC > 1 and adjusted p value < 0.05. Kyoto Encyclopedia of Genes and Genomes (KEGG) pathway enrichment analysis was performed using the Database for Annotation, Visualization and Integrated Discovery (DAVID) (http://david.abcc.ncifcrf.gov/). $\mathrm{P}<0.05$ was used as the threshold criterion.

The experimental data and microarray design have been deposited in the $\mathrm{NCBI}$ Gene Expression Omnibus (GEO) (http://www.ncbi.nlm.nih.gov/geo/) under the accession number GSE115385.

To assess the link between ASAH1 and the three phenotypes (proliferative, invasive, immune) described in Verfaillie et al. ${ }^{33}$, we used the Skin Cutaneous Melanoma (SKCM) dataset from The Cancer Genome Atlas (TCGA). RNA-seq and clinical data were available for 472 patients and downloaded from the TCGA data portal (https://portal.gdc.cancer.gov). RNA-seq data were normalized using the Bioconductor package DESeq2 and log2 transformed. Patients were next classified and assigned to one of the three phenotypes by using molecular marker genes characteristic of each phenotype and by computing a z-score based on the following formula: $z$-score $=$ (expression in sample - mean expression in all samples) $/$ 
standard deviation of expression in all samples. We next computed the median of zscores of each phenotype and then associated the phenotype with the higher value to each patient. We compared the ASAH1 expression in tumor samples, distinguished by the inferred phenotypes. P-values were calculated by the Wilcoxon rank sum test.

\section{Statistical Analysis}

Data are presented as averages $\pm S D$ and were analyzed by two-sided student t-test. A p-value $\leq 0.05$ between the experimental and control groups was considered statistical significant. Degree of similarity analysis of genes with similar patterns of expression to MITF across the melanomas was performed using the Pearson correlation coefficient.

\section{Acknowledgements}

This work was supported by Inserm, La Société Française de Dermatologie and by a grant from INCA (INCA_10573). CP is a fellowship from la Ligue Nationale contre le Cancer. The authors thank Dr M. Sewer (San Diego, USA) for providing the ASAH1 promoter vector. WM3912, WM8, WM3928 and WM3918 human melanoma cell lines were a kind gift from $\mathrm{H}$. Meenhard and G. Zhang (Wistar melanoma Institute, Philadelphia, US).

\section{Declaration of Interests}

The authors declare no conflict of interest. 


\section{Author Contributions}

$\mathrm{CB}, \mathrm{NA}, \mathrm{RB}, \mathrm{TL}$ designed the research, analyzed the results, and wrote the manuscript. GT, SD, JC, PB performed and analysed the immunohistochemistry experiments. NN performed the bioinformatics analysis. JL, DG, CP, CG, KB, VG, PC, SP, BM, performed all the other experiments. 


\section{References}

1 Colombino M, Sini M, Lissia A, De Giorgi V, Stanganelli I, Ayala F et al. Discrepant alterations in main candidate genes among multiple primary melanomas. Journal of translational medicine 2014; $12: 117$.

2 Kemper K, Krijgsman O, Cornelissen-Steijger P, Shahrabi A, Weeber F, Song JY et al. Intra- and inter-tumor heterogeneity in a vemurafenib-resistant melanoma patient and derived xenografts. EMBO molecular medicine 2015; 7 : 1104-1118.

3 Hoek KS, Eichhoff OM, Schlegel NC, Dobbeling U, Kobert N, Schaerer L et al. In vivo switching of human melanoma cells between proliferative and invasive states. Cancer Res 2008; 68: 650-656.

4 Johannessen CM, Johnson LA, Piccioni F, Townes A, Frederick DT, Donahue MK et al. A melanocyte lineage program confers resistance to MAP kinase pathway inhibition. Nature 2013; 504: 138-142.

5 Konieczkowski DJ, Johannessen CM, Abudayyeh O, Kim JW, Cooper ZA, Piris A et al. A Melanoma Cell State Distinction Influences Sensitivity to MAPK Pathway Inhibitors. Cancer discovery 2014.

6 Muller J, Krijgsman O, Tsoi J, Robert L, Hugo W, Song C et al. Low MITF/AXL ratio predicts early resistance to multiple targeted drugs in melanoma. Nature communications 2014; 5: 5712. 
7 Van Allen EM, Foye A, Wagle N, Kim W, Carter SL, McKenna A et al. Successful whole-exome sequencing from a prostate cancer bone metastasis biopsy. Prostate cancer and prostatic diseases 2014; 17: 23-27.

8 Falletta P, Sanchez-Del-Campo L, Chauhan J, Effern M, Kenyon A, Kershaw CJ et al. Translation reprogramming is an evolutionarily conserved driver of phenotypic plasticity and therapeutic resistance in melanoma. Genes Dev 2017; 31: 18-33.

9 Riesenberg S, Groetchen A, Siddaway R, Bald T, Reinhardt J, Smorra D et al. MITF and c-Jun antagonism interconnects melanoma dedifferentiation with pro-inflammatory cytokine responsiveness and myeloid cell recruitment. Nature communications 2015; 6: 8755.

10 Bertolotto C, Lesueur F, Giuliano S, Strub T, de Lichy M, Bille K et al. A SUMOylation-defective MITF germline mutation predisposes to melanoma and renal carcinoma. Nature 2011; 480: 94-98.

11 Bressac de-Paillerets B, Lesueur F, Bertolotto C. A germline oncogenic MITF mutation and tumor susceptibility. European journal of cell biology 2013.

12 Bonet C, Luciani F, Ottavi JF, Leclerc J, Jouenne FM, Boncompagni M et al. Deciphering the Role of Oncogenic MITFE318K in Senescence Delay and Melanoma Progression. J Natl Cancer Inst 2017; 109. 
13 Yokoyama S, Woods SL, Boyle GM, Aoude LG, MacGregor S, Zismann V et al. A novel recurrent mutation in MITF predisposes to familial and sporadic melanoma. Nature 2011; 480: 99-103.

14 Carreira S, Goodall J, Denat L, Rodriguez M, Nuciforo P, Hoek KS et al. Mitf regulation of Dia1 controls melanoma proliferation and invasiveness. Genes Dev 2006; 20: 3426-3439.

15 Cheli Y, Ohanna M, Ballotti R, Bertolotto C. Fifteen-year quest for microphthalmia-associated transcription factor target genes. Pigment Cell Melanoma Res 2010; 23: 27-40.

16 Cheli Y, Giuliano S, Botton T, Rocchi S, Hofman V, Hofman P et al. Mitf is the key molecular switch between mouse or human melanoma initiating cells and their differentiated progeny. Oncogene 2011; 30: 2307-2318.

17 Cheli Y, Giuliano S, Fenouille N, Allegra M, Hofman V, Hofman P et al. Hypoxia and MITF control metastatic behaviour in mouse and human melanoma cells. Oncogene 2012; 31: 2461-2470.

18 Cairns RA, Harris IS, Mak TW. Regulation of cancer cell metabolism. Nat Rev Cancer 2011; 11: 85-95. 
19 Warburg O. On respiratory impairment in cancer cells. Science 1956; 124 : 269-270.

20 Ganapathy-Kanniappan S, Geschwind JF. Tumor glycolysis as a target for cancer therapy: progress and prospects. Molecular cancer 2013; 12: 152.

21 Haq R, Shoag J, Andreu-Perez P, Yokoyama S, Edelman H, Rowe GC et al. Oncogenic BRAF regulates oxidative metabolism via PGC1alpha and MITF. Cancer Cell 2013; 23: 302-315.

22 Vazquez F, Lim JH, Chim H, Bhalla K, Girnun G, Pierce K et al. PGC1alpha expression defines a subset of human melanoma tumors with increased mitochondrial capacity and resistance to oxidative stress. Cancer Cell 2013; 23: 287-301.

23 Currie E, Schulze A, Zechner R, Walther TC, Farese RV, Jr. Cellular fatty acid metabolism and cancer. Cell metabolism 2013; 18: 153-161.

24 Morad SA, Cabot MC. Ceramide-orchestrated signalling in cancer cells. Nat Rev Cancer 2013; 13: 51-65.

25 Ferlinz K, Kopal G, Bernardo K, Linke T, Bar J, Breiden B et al. Human acid ceramidase: processing, glycosylation, and lysosomal targeting. J Biol Chem 2001; 276: 35352-35360. 
26 Rambow F, Job B, Petit V, Gesbert F, Delmas V, Seberg H et al. New Functional Signatures for Understanding Melanoma Biology from Tumor Cell Lineage-Specific Analysis. Cell reports 2015; 13: 840-853.

27 Shtraizent N, Eliyahu E, Park JH, He X, Shalgi R, Schuchman EH. Autoproteolytic cleavage and activation of human acid ceramidase. J Biol Chem 2008; 283: 11253-11259.

28 Zhou J, Tawk M, Tiziano FD, Veillet J, Bayes M, Nolent F et al. Spinal muscular atrophy associated with progressive myoclonic epilepsy is caused by mutations in ASAH1. Am J Hum Genet 2012; 91: 5-14.

29 Bedia C, Casas J, Garcia V, Levade T, Fabrias G. Synthesis of a novel ceramide analogue and its use in a high-throughput fluorogenic assay for ceramidases. Chembiochem : a European journal of chemical biology 2007; 8: $642-648$

30 Strub T, Giuliano S, Ye T, Bonet C, Keime C, Kobi D et al. Essential role of microphthalmia transcription factor for DNA replication, mitosis and genomic stability in melanoma. Oncogene 2011; 30: 2319-2332.

31 Ohanna M, Cerezo M, Nottet N, Bille K, Didier R, Beranger G et al. Pivotal role of NAMPT in the switch of melanoma cells toward an invasive and drugresistant phenotype. Genes Dev 2018; 32: 448-461. 
32 Walkley SU, Vanier MT. Secondary lipid accumulation in lysosomal disease. Biochim Biophys Acta 2009; 1793: 726-736.

33 Verfaillie A, Imrichova H, Atak ZK, Dewaele M, Rambow F, Hulselmans G et al. Decoding the regulatory landscape of melanoma reveals TEADS as regulators of the invasive cell state. Nature communications 2015; 6: 6683.

34 Caramel J, Papadogeorgakis E, Hill L, Browne GJ, Richard G, Wierinckx A et al. A Switch in the Expression of Embryonic EMT-Inducers Drives the Development of Malignant Melanoma. Cancer Cell 2013; 24: 466-480.

35 Realini N, Palese F, Pizzirani D, Pontis S, Basit A, Bach A et al. Acid Ceramidase in Melanoma: EXPRESSION, LOCALIZATION, AND EFFECTS OF PHARMACOLOGICAL INHIBITION. J Biol Chem 2016; 291: 2422-2434.

36 Lucki N, Sewer MB. The CAMP-responsive element binding protein (CREB) regulates the expression of acid ceramidase (ASAH1) in H295R human adrenocortical cells. Biochim Biophys Acta 2009; 1791: 706-713.

37 Lucki NC, Sewer MB. Genistein stimulates MCF-7 breast cancer cell growth by inducing acid ceramidase (ASAH1) gene expression. J Biol Chem 2011; 286: 19399-19409.

38 Don AS, Lim XY, Couttas TA. Re-configuration of sphingolipid metabolism by oncogenic transformation. Biomolecules 2014; 4: 315-353. 
39 Truman JP, Garcia-Barros M, Obeid LM, Hannun YA. Evolving concepts in cancer therapy through targeting sphingolipid metabolism. Biochim Biophys Acta 2014; 1841: 1174-1188.

40 Cheng JC, Bai A, Beckham TH, Marrison ST, Yount CL, Young $\mathrm{K}$ et al. Radiation-induced acid ceramidase confers prostate cancer resistance and tumor relapse. J Clin Invest 2013; 123: 4344-4358.

41 Elojeimy S, Liu X, McKillop JC, El-Zawahry AM, Holman DH, Cheng JY et al. Role of acid ceramidase in resistance to FasL: therapeutic approaches based on acid ceramidase inhibitors and FasL gene therapy. Molecular therapy : the journal of the American Society of Gene Therapy 2007; 15: 1259-1263.

42 Mahdy AE, Cheng JC, Li J, Elojeimy S, Meacham WD, Turner LS et al. Acid ceramidase upregulation in prostate cancer cells confers resistance to radiation: AC inhibition, a potential radiosensitizer. Molecular therapy : the journal of the American Society of Gene Therapy 2009; 17: 430-438.

43 Ruckhaberle E, Holtrich U, Engels K, Hanker L, Gatje R, Metzler D et al. Acid ceramidase 1 expression correlates with a better prognosis in ER-positive breast cancer. Climacteric : the journal of the International Menopause Society 2009; 12: 502-513. 
44 Sanger N, Ruckhaberle E, Gyorffy B, Engels K, Heinrich T, Fehm T et al. Acid ceramidase is associated with an improved prognosis in both DCIS and invasive breast cancer. Molecular oncology 2015; 9: 58-67.

45 Park JH, Schuchman EH. Acid ceramidase and human disease. Biochim Biophys Acta 2006; 1758: 2133-2138.

46 Bonet C, Giuliano S, Ohanna M, Bille K, Allegra M, Lacour JP et al. Aurora B is regulated by the mitogen-activated protein kinase/extracellular signalregulated kinase (MAPK/ERK) signaling pathway and is a valuable potential target in melanoma cells. J Biol Chem 2012; 287: 29887-29898.

47 Giuliano S, Cheli Y, Ohanna M, Bonet C, Beuret L, Bille $\mathrm{K}$ et al. Microphthalmia-associated transcription factor controls the DNA damage response and a lineage-specific senescence program in melanomas. Cancer Res 2010; 70: 3813-3822.

48 Lai M, Realini N, La Ferla M, Passalacqua I, Matteoli G, Ganesan A et al. Complete Acid Ceramidase ablation prevents cancer-initiating cell formation in melanoma cells. Scientific reports 2017; 7: 7411.

49 Ohanna M, Giuliano S, Bonet C, Imbert V, Hofman V, Zangari J et al. Senescent cells develop a PARP-1 and nuclear factor-\{kappa\}B-associated secretome (PNAS). Genes Dev 2011; 25: 1245-1261. 
50 Ohanna M, Cheli Y, Bonet C, Bonazzi VF, Allegra M, Giuliano S et al. Secretome from senescent melanoma engages the STAT3 pathway to favor reprogramming of naive melanoma towards a tumor-initiating cell phenotype. Oncotarget 2013; 4: 2212-2224.

51 Gupta PB, Kuperwasser C, Brunet JP, Ramaswamy S, Kuo WL, Gray JW et al. The melanocyte differentiation program predisposes to metastasis after neoplastic transformation. Nat Genet 2005; 37: 1047-1054.

52 Pinner S, Jordan P, Sharrock K, Bazley L, Collinson L, Marais R et al. Intravital imaging reveals transient changes in pigment production and Brn2 expression during metastatic melanoma dissemination. Cancer Res 2009; 69: 7969-7977.

53 Villanueva J, Vultur A, Lee JT, Somasundaram R, Fukunaga-Kalabis M, Cipolla AK et al. Acquired resistance to BRAF inhibitors mediated by a RAF kinase switch in melanoma can be overcome by cotargeting MEK and IGF1R/PI3K. Cancer Cell 2010; 18: 683-695.

54 Widmer DS, Cheng PF, Eichhoff OM, Belloni BC, Zipser MC, Schlegel NC et al. Systematic classification of melanoma cells by phenotype-specific gene expression mapping. Pigment Cell Melanoma Res 2012; 25: 343-353. 
55 Reinhardt J, Landsberg J, Schmid-Burgk JL, Ramis BB, Bald T, Glodde N et al. MAPK Signaling and Inflammation Link Melanoma Phenotype Switching to Induction of CD73 during Immunotherapy. Cancer Res 2017; 77: 4697-4709.

56 Webster MR, Xu M, Kinzler KA, Kaur A, Appleton J, O'Connell MP et al. Wnt5A promotes an adaptive, senescent-like stress response, while continuing to drive invasion in melanoma cells. Pigment Cell Melanoma Res 2015; 28: 184-195.

57 Canel M, Serrels A, Frame MC, Brunton VG. E-cadherin-integrin crosstalk in cancer invasion and metastasis. J Cell Sci 2013; 126: 393-401.

58 Vesuna $\mathrm{F}$, van Diest $\mathrm{P}$, Chen $\mathrm{JH}$, Raman $\mathrm{V}$. Twist is a transcriptional repressor of E-cadherin gene expression in breast cancer. Biochem Biophys Res Commun 2008; 367: 235-241.

59 Wang F, Van Brocklyn JR, Edsall L, Nava VE, Spiegel S. Sphingosine-1phosphate inhibits motility of human breast cancer cells independently of cell surface receptors. Cancer Res 1999; 59: 6185-6191.

60 Desch A, Strozyk EA, Bauer AT, Huck V, Niemeyer V, Wieland T et al. Highly invasive melanoma cells activate the vascular endothelium via an MMP2/integrin alphavbeta5-induced secretion of VEGF-A. Am J Pathol 2012; 181: 693-705. 
61 Fane ME, Chhabra Y, Hollingsworth DE, Simmons JL, Spoerri L, Oh TG et al. NFIB Mediates BRN2 Driven Melanoma Cell Migration and Invasion Through Regulation of EZH2 and MITF. EBioMedicine 2017; 16: 63-75.

62 Goodall J, Carreira S, Denat L, Kobi D, Davidson I, Nuciforo P et al. Brn-2 represses microphthalmia-associated transcription factor expression and marks a distinct subpopulation of microphthalmia-associated transcription factor-negative melanoma cells. Cancer Res 2008; 68: 7788-7794.

63 Larribere L, Hilmi C, Khaled M, Gaggioli C, Bille K, Auberger P et al. The cleavage of microphthalmia associated transcription factor, MITF, by caspases plays an essential role in melanocyte and melanoma cell apoptosis. Genes Dev 2005; 19: 1980-1985.

64 Merrill AH, Jr., Sullards MC, Allegood JC, Kelly S, Wang E. Sphingolipidomics: high-throughput, structure-specific, and quantitative analysis of sphingolipids by liquid chromatography tandem mass spectrometry. Methods 2005; 36: $207-$ 224. 


\section{Figure legends}

\section{Figure 1: Acid ceramidase ASAH1 is expressed in melanoma cells}

(A) Heatmap showing the expression of genes in the invasive and proliferative cells. The data were analysed using the Morpheus software (https://software.broadinstitute.org/morpheus/). A relative color scheme based on the minimum and maximum values in each row was used to convert values to color. The relative levels are indicated by varying color intensity (low-blue, high-red).

(B) Box plots showing the relative ASAH1 mRNA across the different tumor types, extracted from in the $\mathrm{NCl} 60$ lines datasets.

(C) Co-expression of MITF and ASAH1 in melanoma from the TCGA database. Gene expression data were $\log _{2}$ transformed. Pearson's correlation coefficient, used to measure similarity between gene profiles, is shown.

(D) Immunoblot to ASAH1 of total cell extracts isolated from the indicated melanoma cell lines with a non-commercial antibody showing the proform (55 kDa), the $\beta$ (40 $\mathrm{kDa})$ and $\alpha(14 \mathrm{kDa})$ active subunits. Detection of ERK2 serves as a loading control.

(E) Immunoblot to MITF of total cell extracts isolated from the indicated melanoma cell lines. MITF is detected as a doublet of $55-72 \mathrm{kDa}$. Detection of ERK2 serves as a loading control.

(F) ASAH1 activity in a panel of melanoma cells. Values represent mean + SD of two independent experiments.

(G) Cellular lipids were extracted, and sphingolipid concentration was determined by LC/MS. Data represent the quantity of sphingosine $(\mathrm{SPH})$ in $\mathrm{ng} / \mathrm{mg}$ proteins and are the means $+\mathrm{SD}$ of two independent determinations. 


\section{Figure 2: Expression of MITF and ASAH1 in human melanoma samples}

(A-B) IHC analyses of MITF and ASAH1 expression at superficial and deep sites of primary melanoma sample. The images exemplify correlation between MITF and ASAH1 expression within the lesions. Magnification 4x, scale bar: $500 \mu \mathrm{m}$ and magnification 20x, scale bar: $100 \mu \mathrm{m}$.

(C) IHC analyses of MITF and ASAH1 expression in a cutaneous metastatic melanoma sample. Magnification 4x, scale bar: $500 \mu \mathrm{m}$ and magnification 20x, scale bar: $100 \mu \mathrm{m}$.

\section{Figure 3: ASAH1 is a direct target of the MITF transcription factor}

(A) mRNA expression of MITF and two of its target genes (Tyrosinase and MLANA) and ASAH1 in 501mel melanoma cell lines determined by qRT-PCR. Values represent mean $+S D$ of three independent experiments performed in triplicate. ${ }^{* * *} p<0.001$.

(B) Immunoblot to MITF and ASAH1 of 501 mel melanoma cells transfected with control or Mitf-specific siRNA. Detection of ERK2 serves as a loading control.

(C) ASAH1 activity of of $501 \mathrm{mel}$ melanoma cells transfected with control or Mitfspecific siRNA. Values represent mean + SD of three independent experiments. ${ }^{* *} p<0.01$.

(D) Immunoblot to MITF of Mel-ST melanocyte cells transduced with an empty adenoviral vector or a vector encoding MITF.

(E) mRNA expression of ASAH1 in Mel-ST melanocyte cells transduced with an empty adenoviral vector or a vector encoding MITF determined by qRT-PCR. Values represent mean $+S D$ of three independent experiments performed in triplicate. ${ }^{* *} p<0.01$. 
(F) ASAH1 activity in Mel-ST melanocyte cells transduced with an empty adenoviral vector or a vector encoding MITF. Values represent mean + SD of three independent experiments performed in triplicate. ${ }^{*} p<0.05$.

(G) ChIP-seq profile showing significant MITF binding peaks in the ASAH1 genomic region (black arrowheads). The sequence under peak shows the presence of MITFbinding sites. The scale bar indicates the size of the genomic region in kilobases (kB). The H3K27ac and H3K4me3 tracks associated with active transcription, and the H3K27me3 track associated with the repression of transcription were obtained in control $501 \mathrm{mel}$ cells ${ }^{31}$ and were aligned with the ChiP-seq profile of MITF ${ }^{30}$.

(H) a, Cell count of 501 mel melanoma cells infected with an empty vector or a vector encoding ASAH1 and transfected with control or MITF siRNA. Representative micrographs are shown. Values represent mean + SD of three independent experiments. ${ }^{* *} \mathrm{p}<0.001$. b, Immunoblot of 501 mel melanoma cells infected with an empty vector or a vector encoding ASAH1 and transfected with control or MITF siRNA.

\section{Figure 4: ASAH1 controls melanoma cell proliferation and motile features}

(A) ASAH1 mRNA expression in melanoma cells transfected with control or two different ASAH1 (ASAH1\#1 and ASAH1\#2) or MITF siRNA. Values represent mean $+\mathrm{SD}$ of three independent experiments performed in triplicate. ${ }^{* *} \mathrm{p}<0.01 ;{ }^{* * *} \mathrm{p}<0.001$. (B) Boyden chambers experiments of melanoma cells transfected with a control or two different ASAH1 siRNA (ASAH1\#1 and ASAH1\#2) or with a MITF siRNA. Representative images are shown. Values represent mean + SD of three independent experiments. ${ }^{*} p<0.05,{ }^{* *} p<0.01$ and ${ }^{* * *} p<0.001$. 
(C) Boyden chambers experiments. 501 mel melanoma cells were transfected for 48 hrs with ASAH1 or MITF siRNA before being exposed to $3 \mu \mathrm{M}$ D-sphingosine (DSPH) for 5 hrs. Values represent mean + SD of three independent experiments. ${ }^{* *} \mathrm{p}<0.01 ;{ }^{* * *} \mathrm{p}<0.001$

(D) Boyden chamber experiments. Invasive melanoma cells were exposed to $3 \mu \mathrm{M}$ D-sphingosine (D-SPH) for 5 hrs. Values represent mean + SD of three independent experiments. ${ }^{* * *} p<0.001$.

\section{Figure 5: ASAH1 controls cell migration via the integrin/FAK cascade}

(A) Top significantly deregulated pathways upon ASAH1 downregulation in three different melanoma cell lines (501mel, WM3912, WM8) transfected with control or ASAH1 SIRNA.

(B) Immunoblot to total and phosphorylated FAK (Y397) after ASAH1 downregulation (48hrs) compared to control siRNA.

(C) Confocal microscopy images of phosphorylated FAK (Y397) localization after ASAH1 down-regulation (48hrs) compared to control siRNA.

(D) Immunoblot to total and phosphorylated FAK (Y397), ASAH1 and HSP90 (loading control) in WM3918 with forced expression of ASAH1 compared to the parental cells.

(E) Immunoblot to ITG $\beta 5$, ITG $\alpha$ V and HSP90 (loading control) in 501mel, WM3912 and WM8 melanoma cells transfected with a control or ASAH1 siRNA.

(F) Immunofluorescence of ITG $\beta 5$ in WM3912.

(G) Boyden chambers experiments with control IgG Ctl) or ITG $\alpha$ V $\beta 5$ neutralizing antibody $(\mathrm{ITG} \alpha \mathrm{V} \beta 5)$. Values represent mean + SD of three independent experiments. ${ }^{* * *} p<0.001$. Representative images are shown. 
A

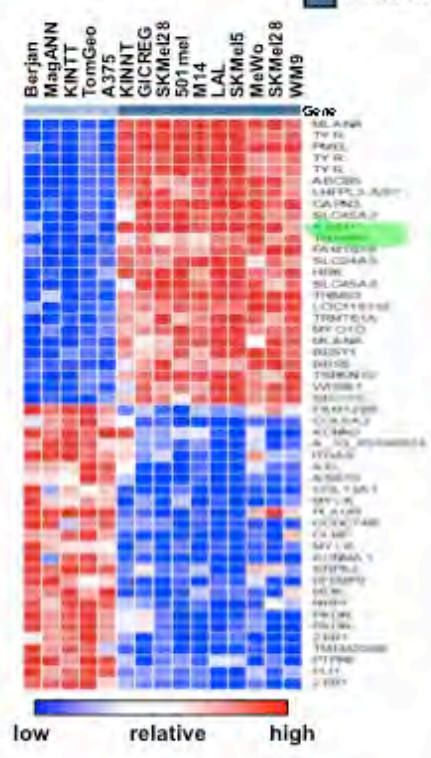

D

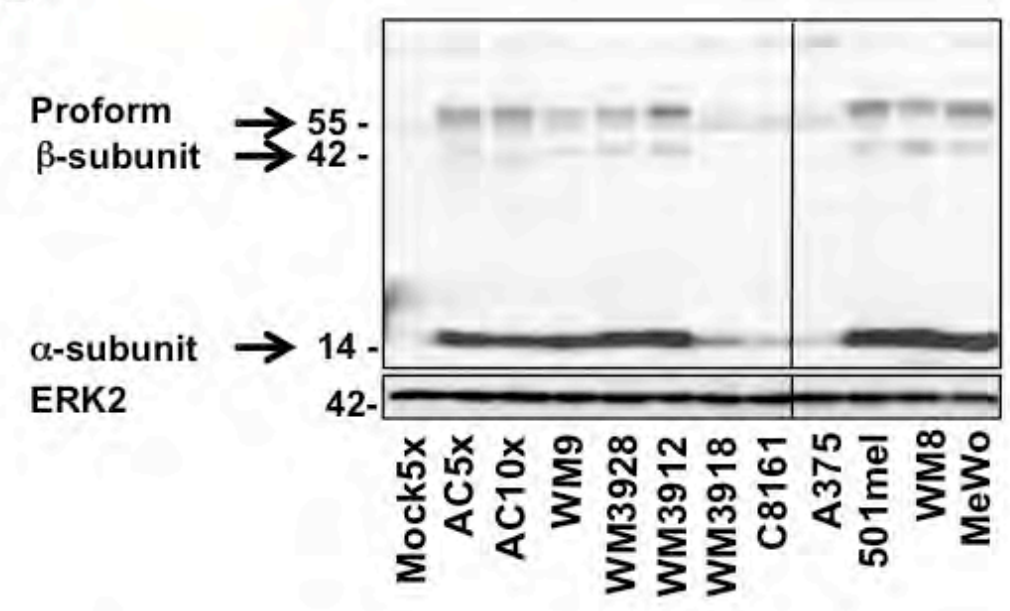

E

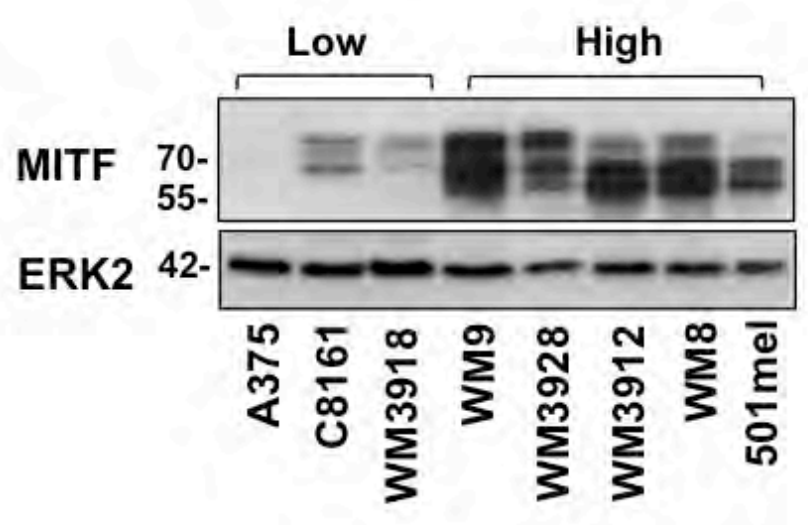

C
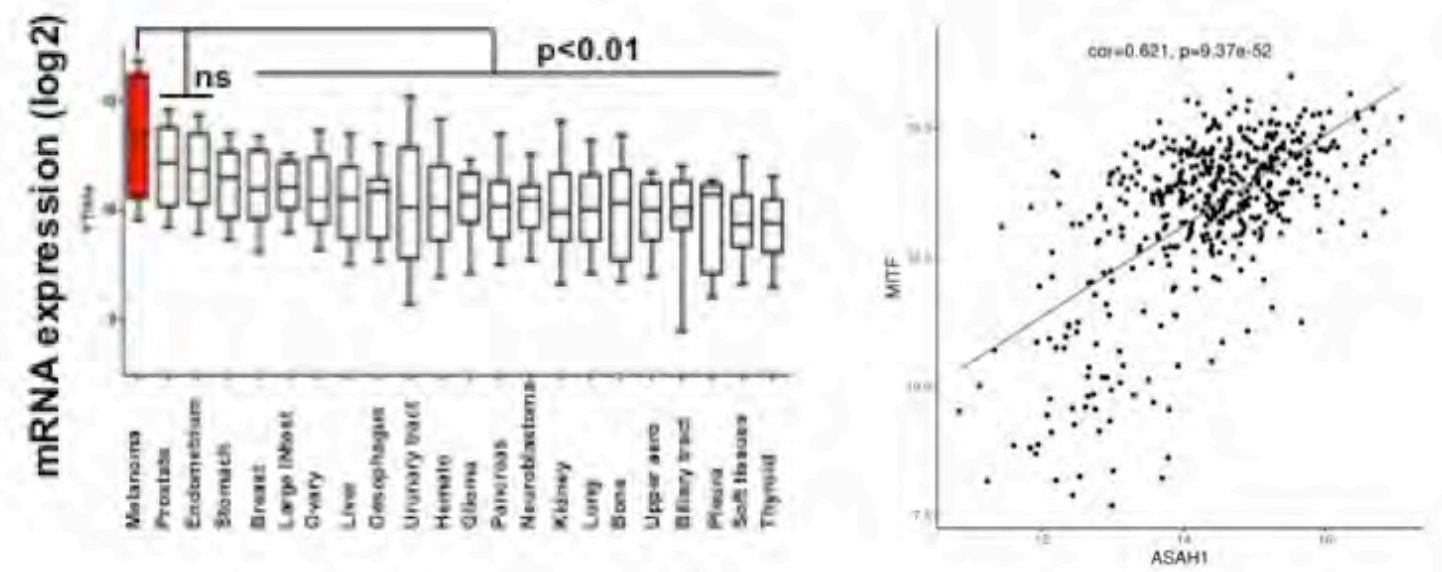

F

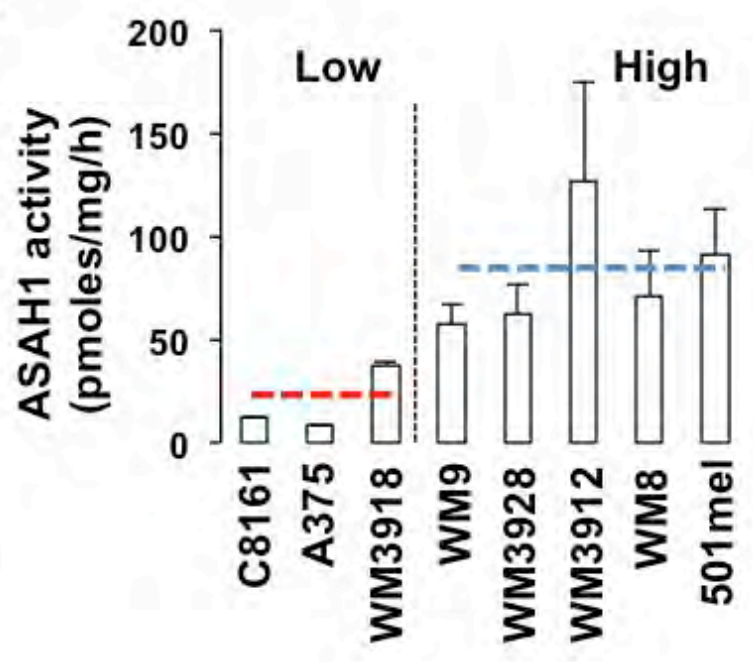

G

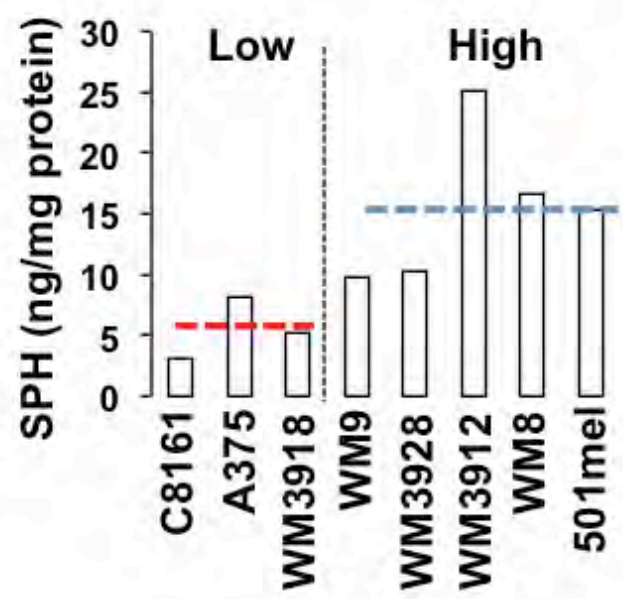


A

B
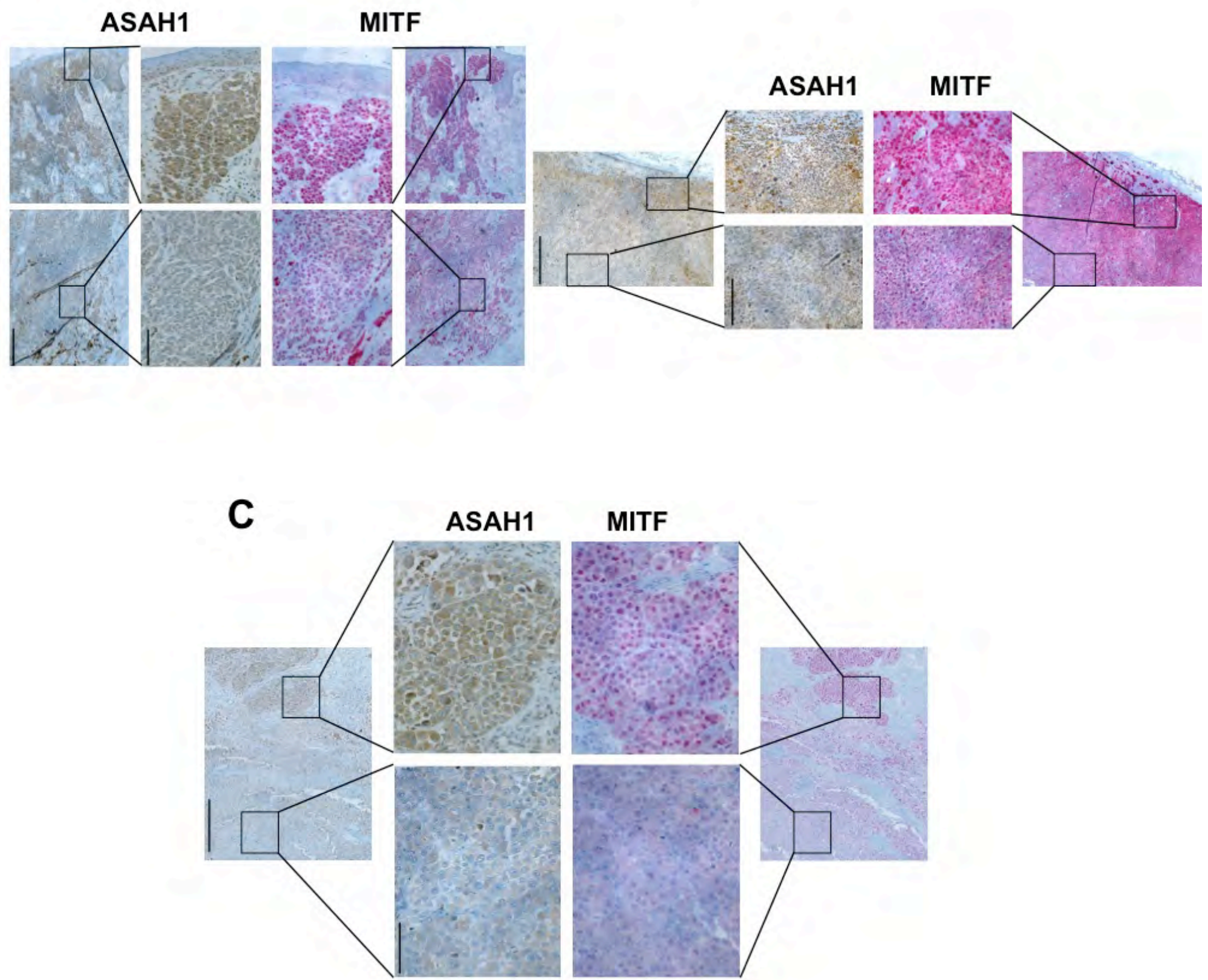
A
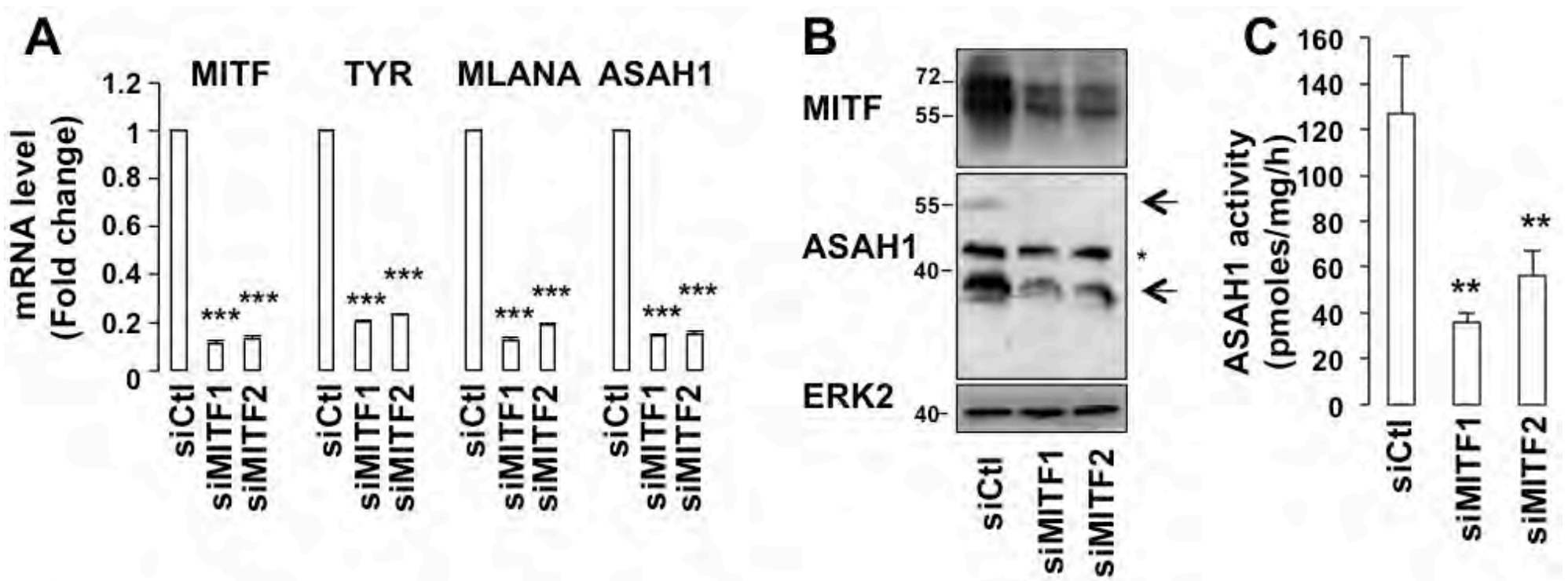

D

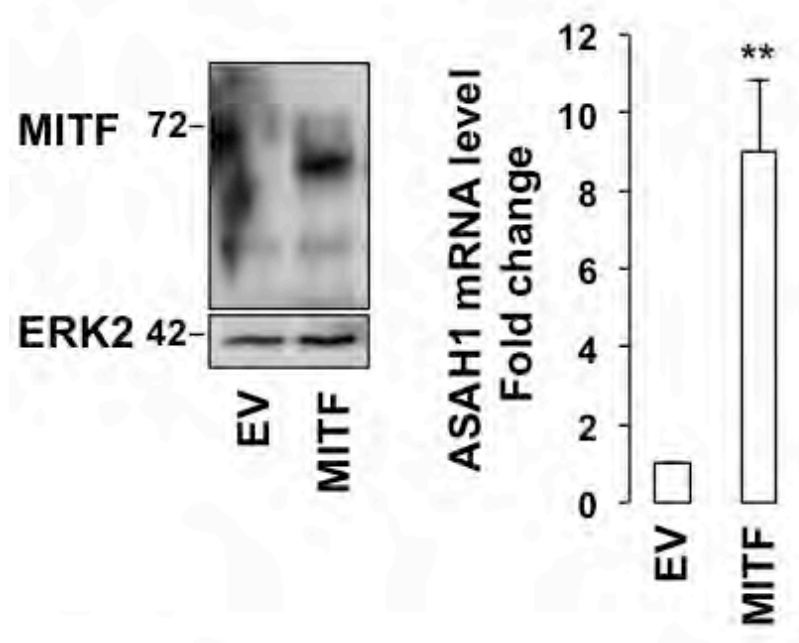

H
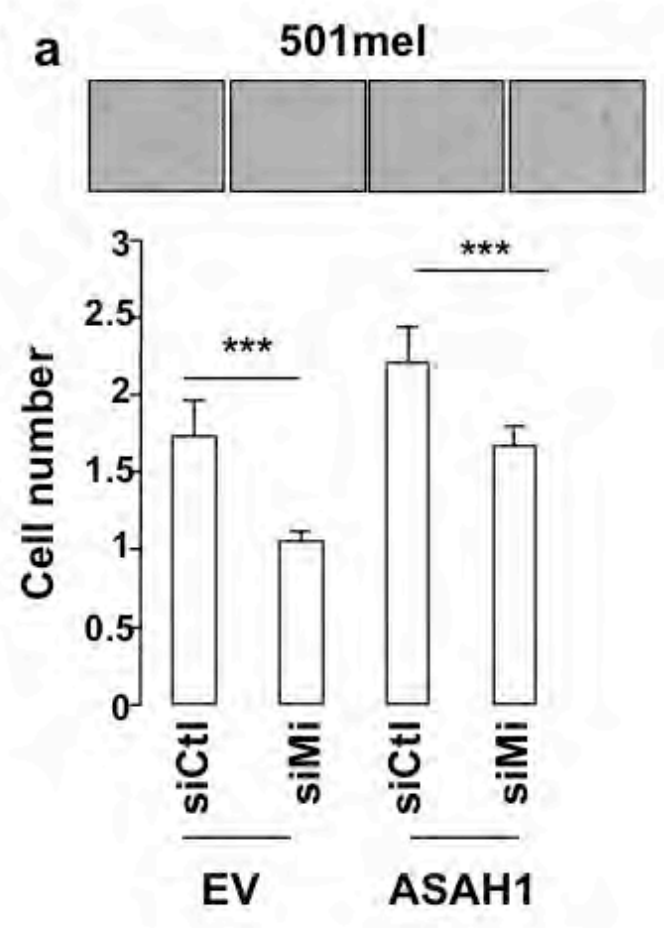

F

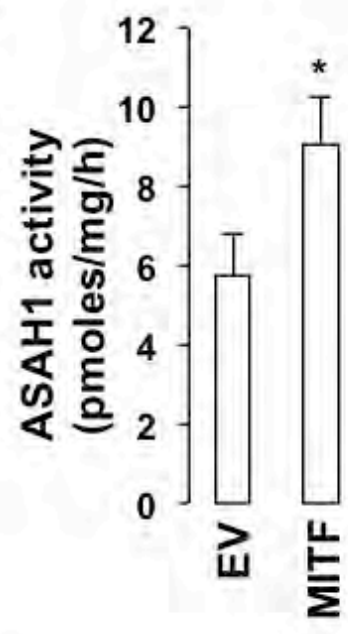

G

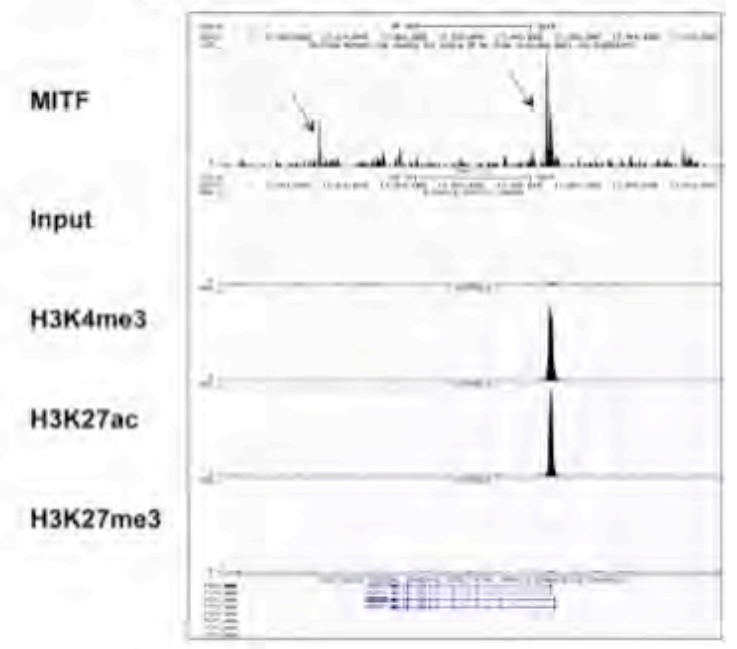

b

$501 \mathrm{mel}$

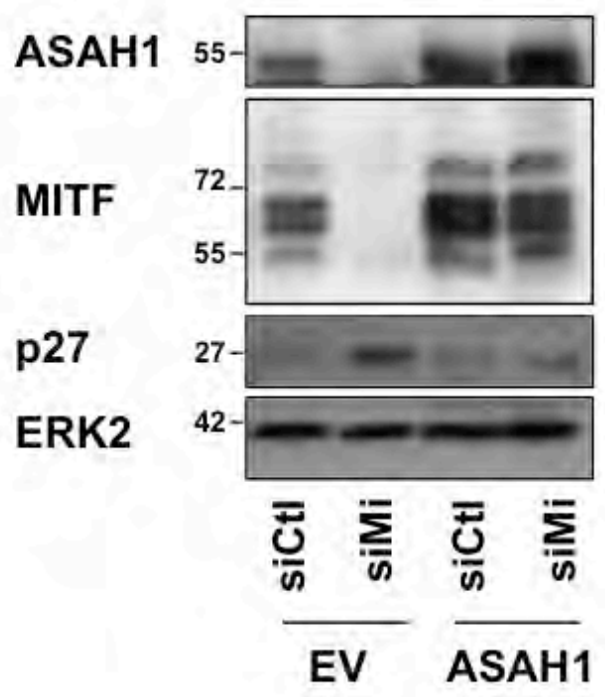


A

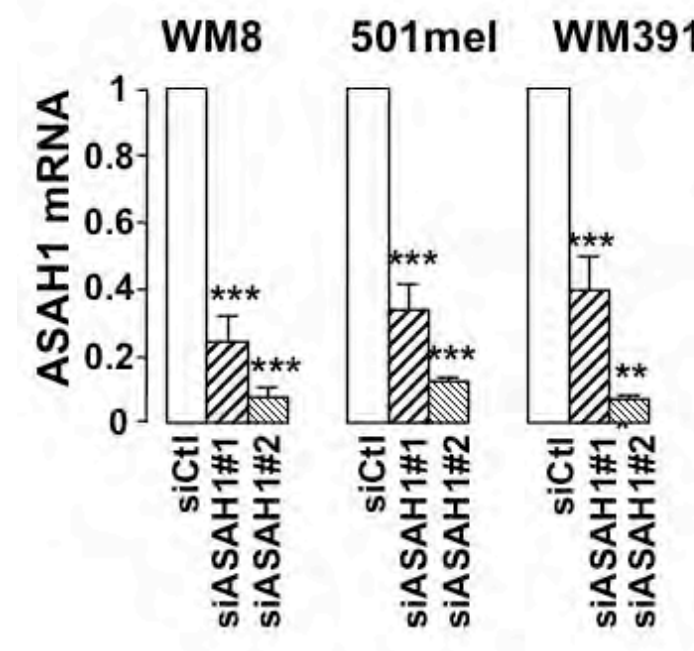

B

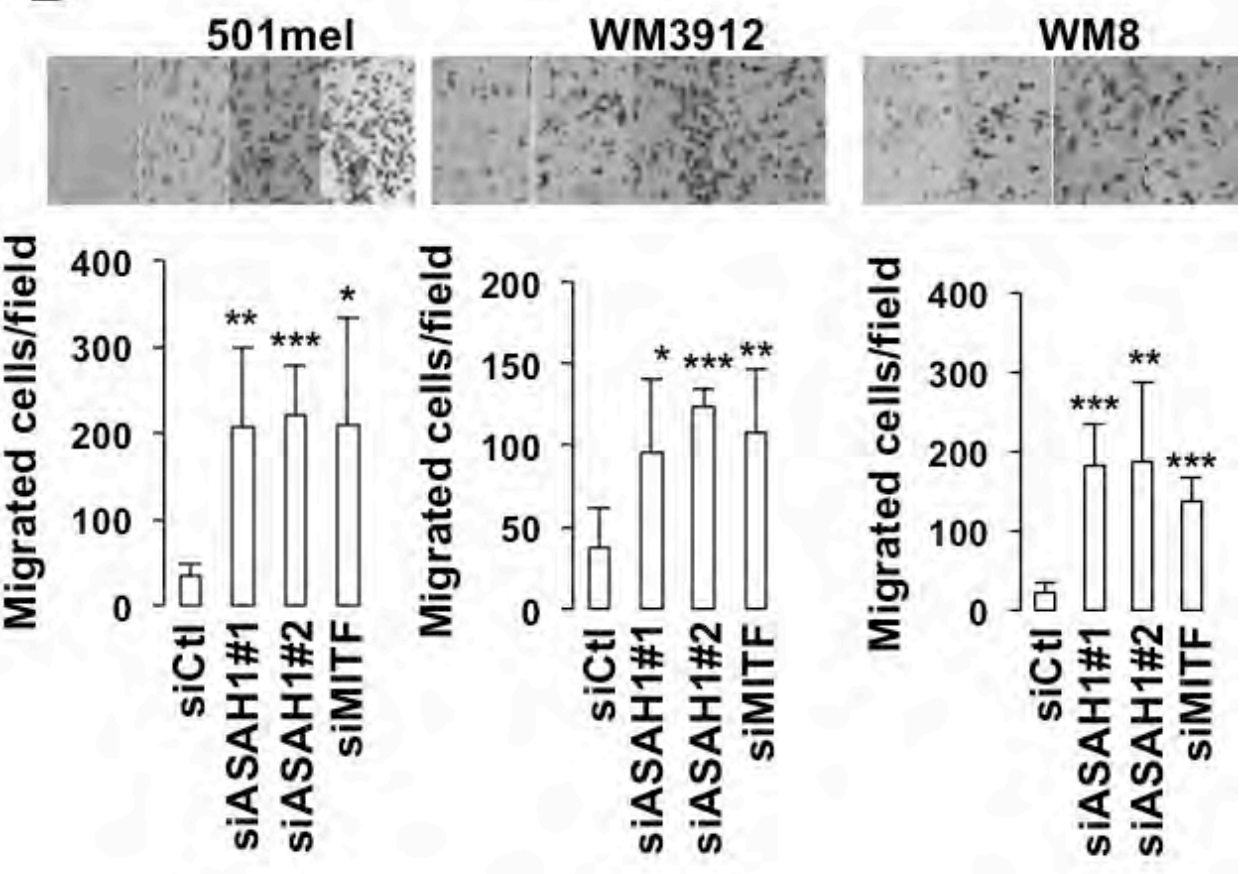

C siCtl SIASAH1 SIMITF
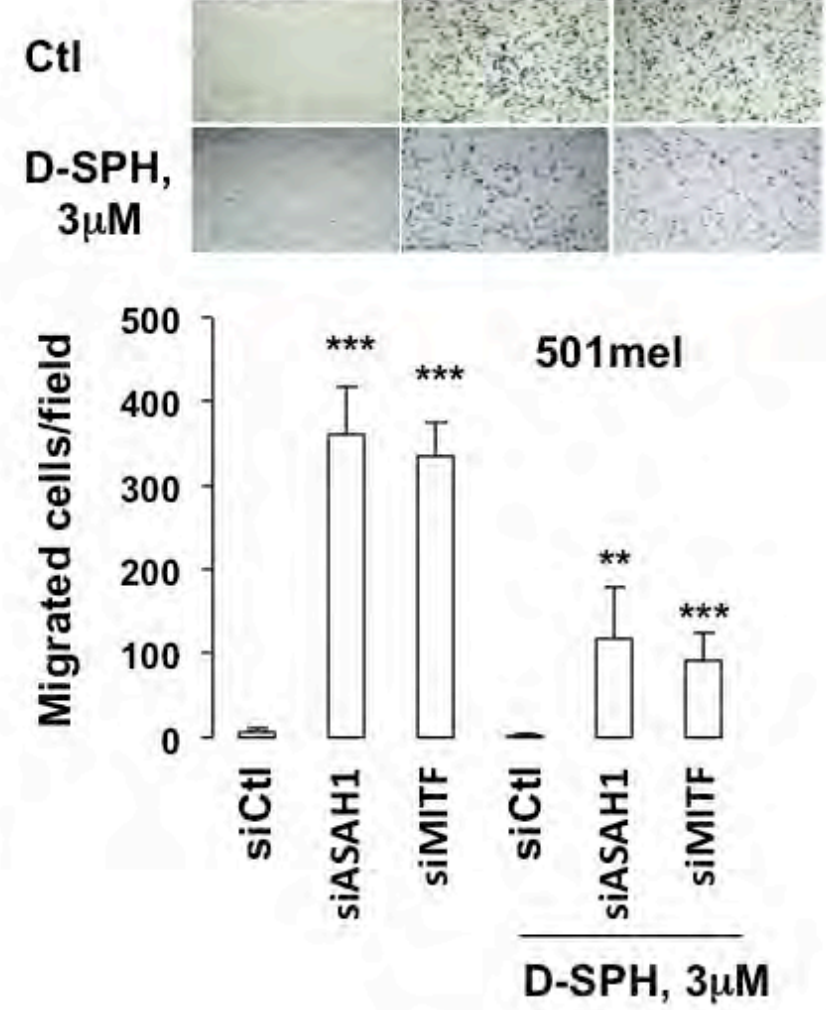

A375 C8161 WM3918

Ctl

D-SPH, $3 \mu \mathrm{M}$

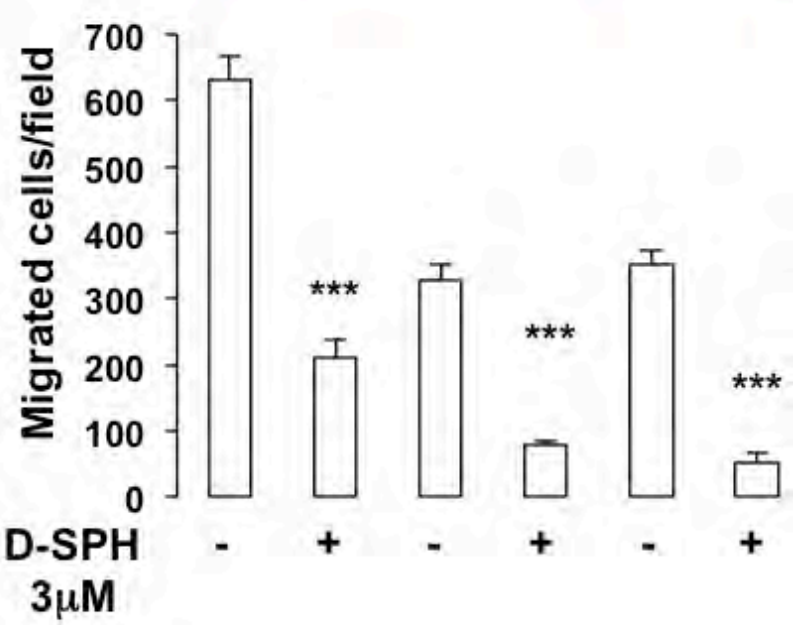




\begin{tabular}{|c|c|c|}
\hline A & $\underline{\text { Terms }}$ & PVALUE \\
\hline & Pathways in cancer & 7.70E-03 \\
\hline & Focal adhesion & $4.50 E-03$ \\
\hline & Adherens junction & $2.60 \mathrm{E}-0$ \\
\hline & Endocytosis & $3.20 \mathrm{E}-02$ \\
\hline & Axon guidance & 7.70E-03 \\
\hline & Viral myocarditis & 1.70E-03 \\
\hline & Cardiomyopathy & 3.00E-02 \\
\hline & ECM-receptor interaction & $5.00 \mathrm{E}-02$ \\
\hline & mTOR signaling pathway & 2.40E-02 \\
\hline & Arginine and proline metabolism & 1.60E-02 \\
\hline 5 & $\begin{array}{l}\text { RNA degradation } \\
\text { Pathogrenic. Esche }\end{array}$ & 8.80E-02 \\
\hline$=$ & & \\
\hline
\end{tabular}

C

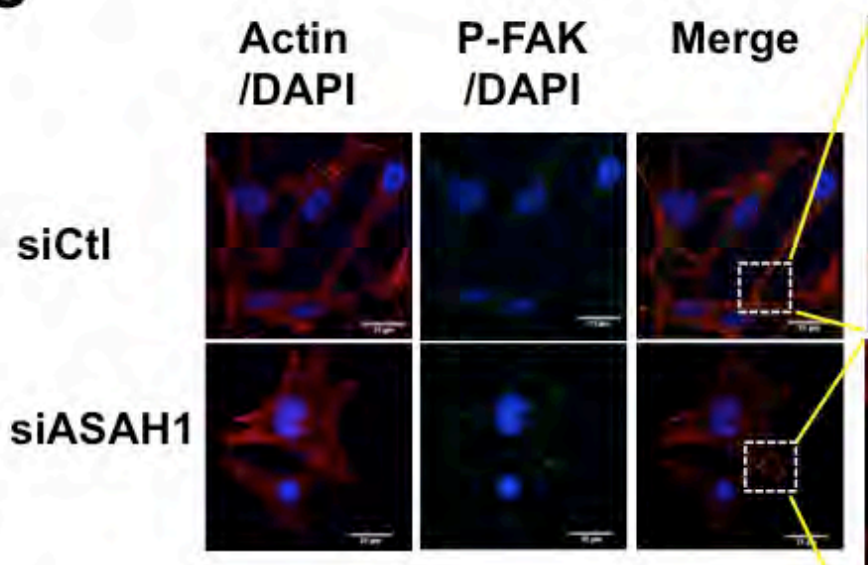

E

$\mathbf{F}$

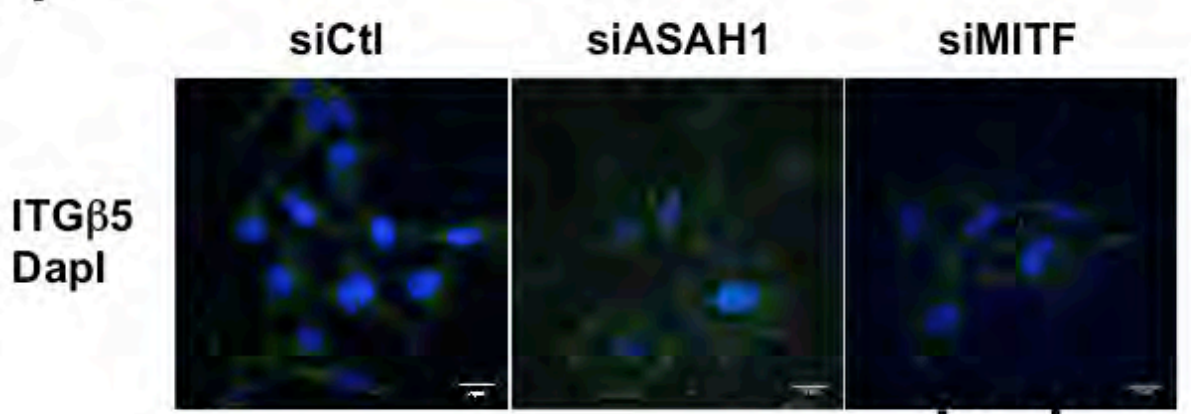

B

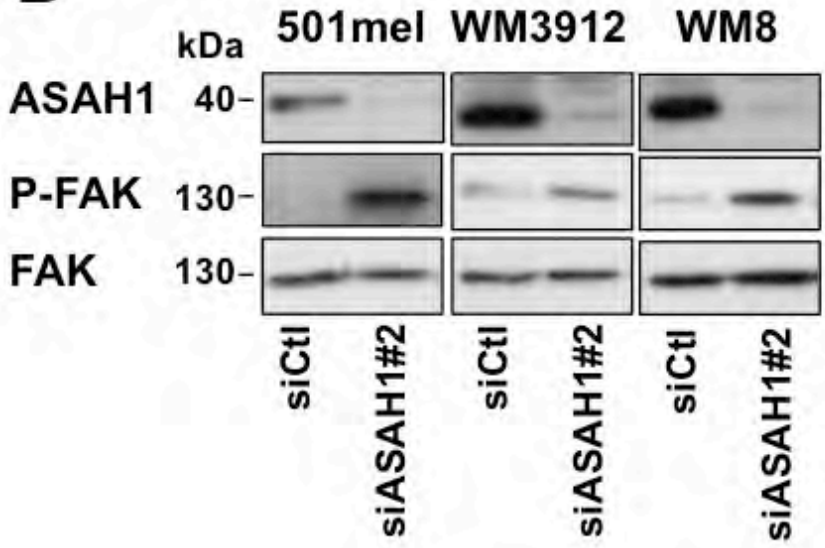

D
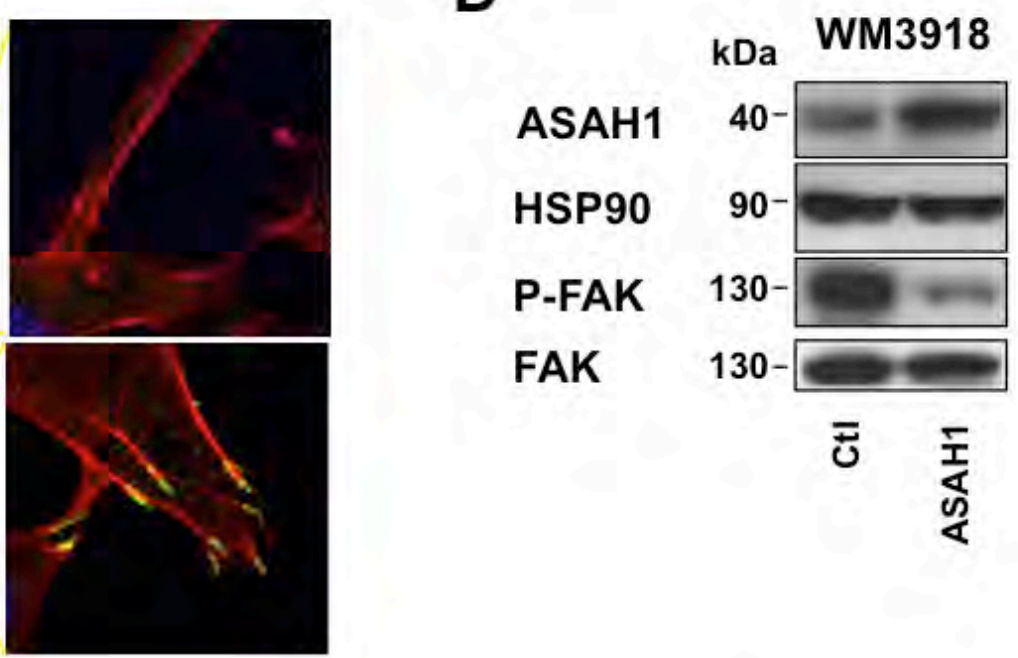

501mel

siCtI SIASAH1 siMITF

Ctl

ITG $\alpha$ V $\beta 5$

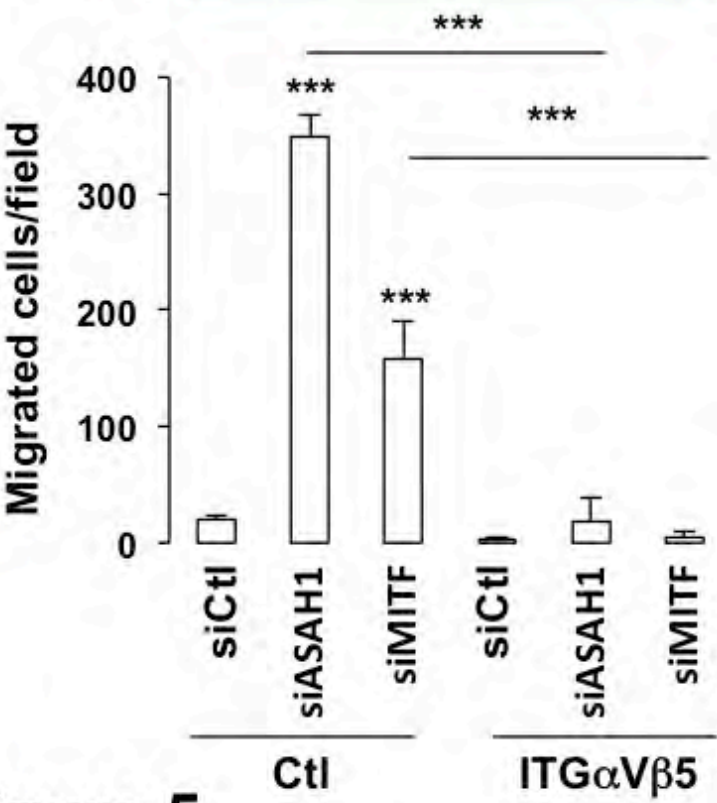


A

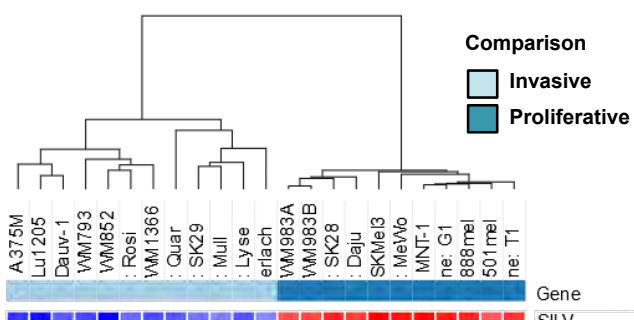

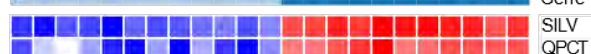
\begin{tabular}{l}
1 \\
\hline
\end{tabular}

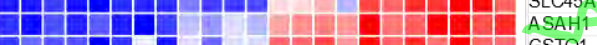
\begin{tabular}{lll}
\hline-12 & & GSTO1 \\
LOC390595
\end{tabular} \begin{tabular}{l}
\hline \\
\hline
\end{tabular} \begin{tabular}{ll|l|l}
10 & -1
\end{tabular}

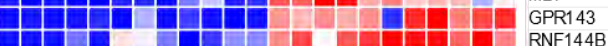
10 H

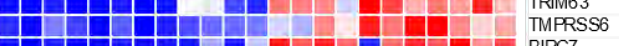

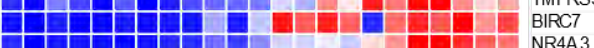

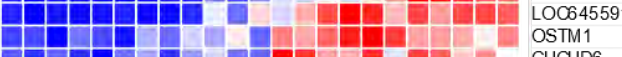

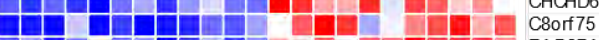

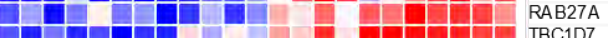
1 -

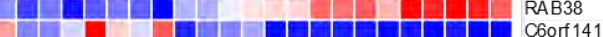

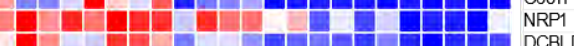
H \begin{tabular}{l|l|l|l|l|l|l|l|l|l|l} 
CNN2 \\
COL13A1
\end{tabular} 1 1 -

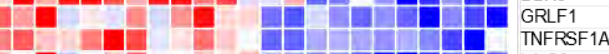

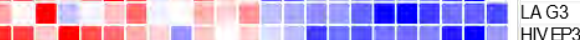
H

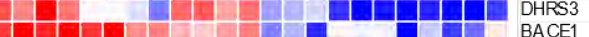

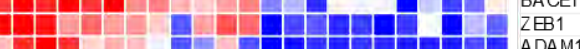

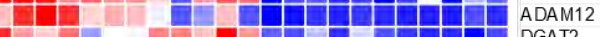

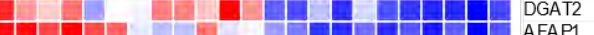
1 - 1 H

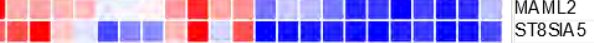

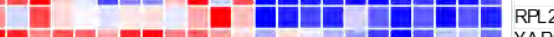
\begin{tabular}{l|l|l|l|l|l|l|} 
YAP1 \\
RRF1
\end{tabular}
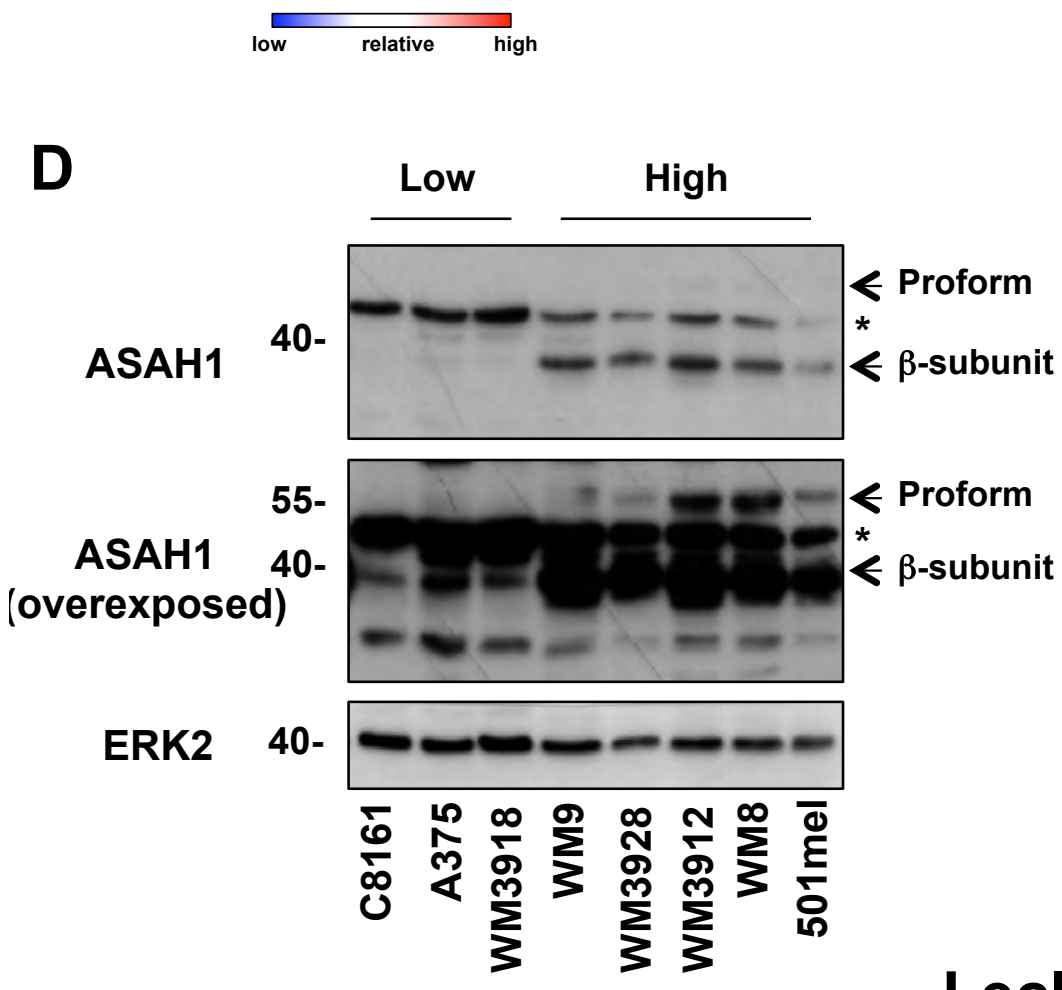
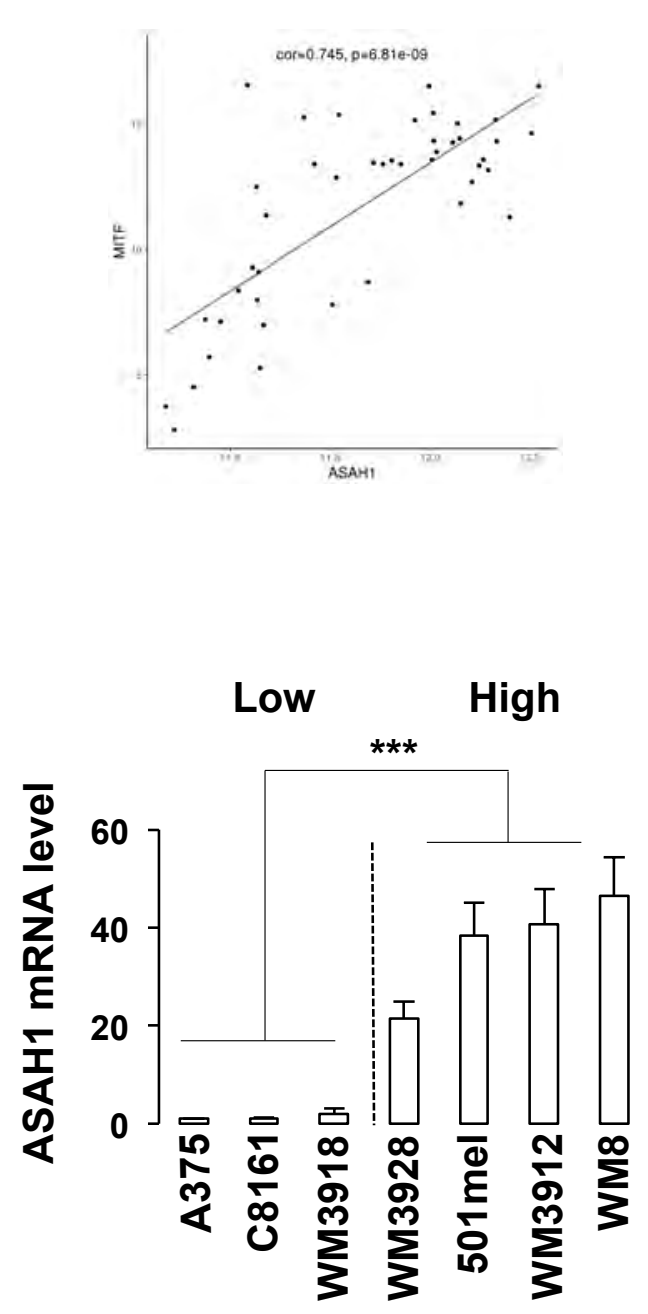

E

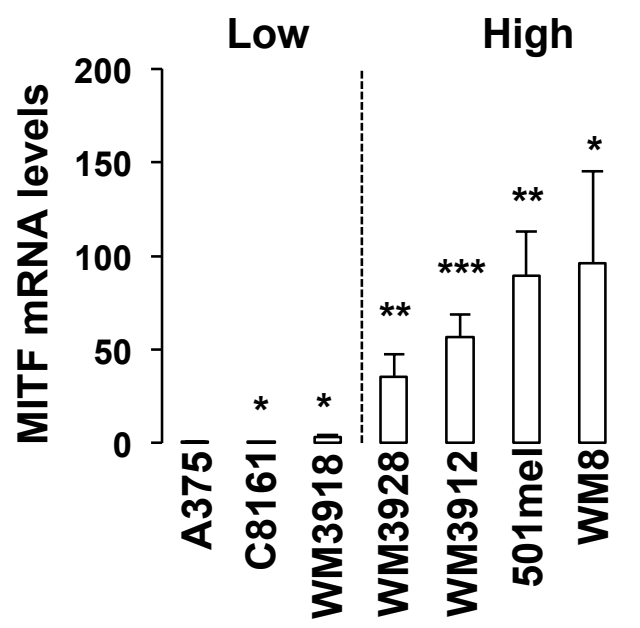



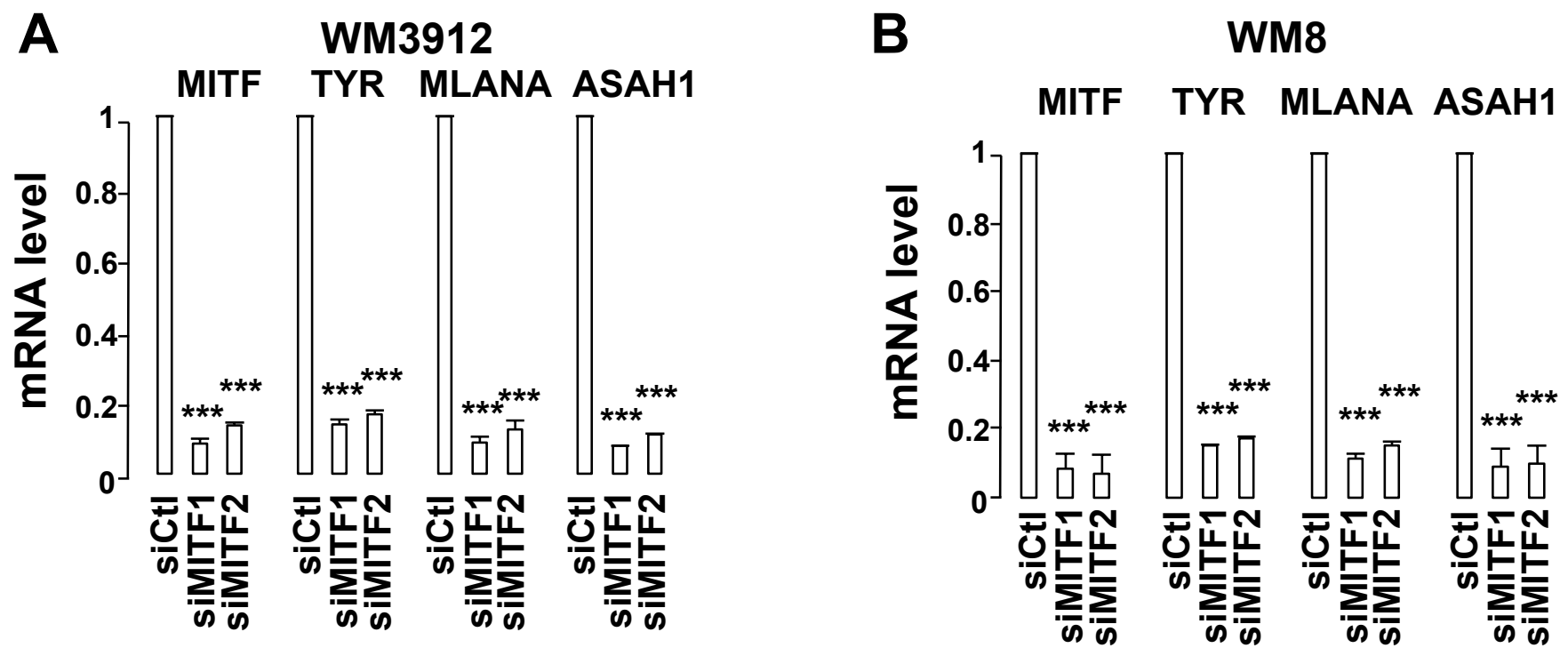

C

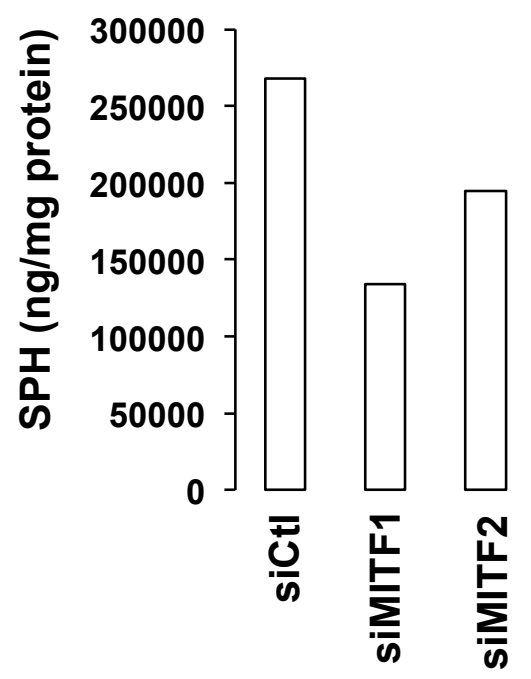

E

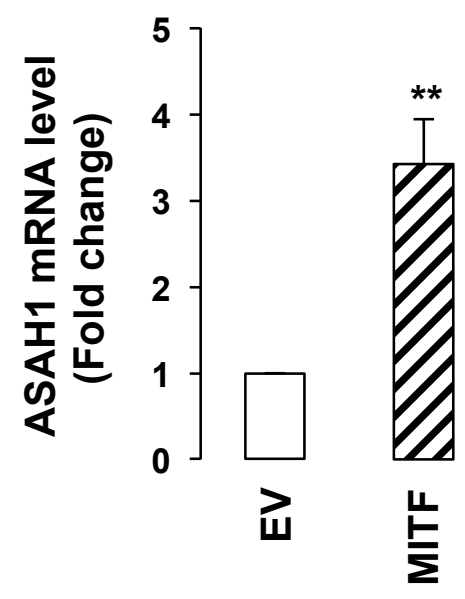

E
F

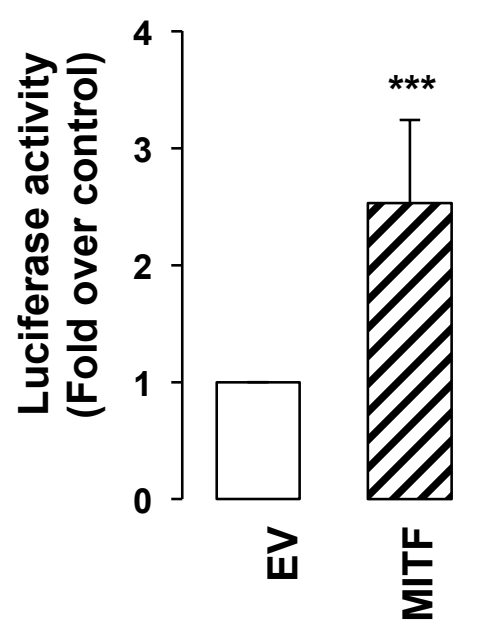

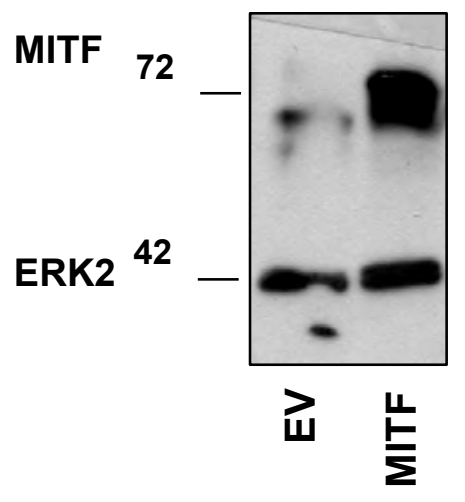

G

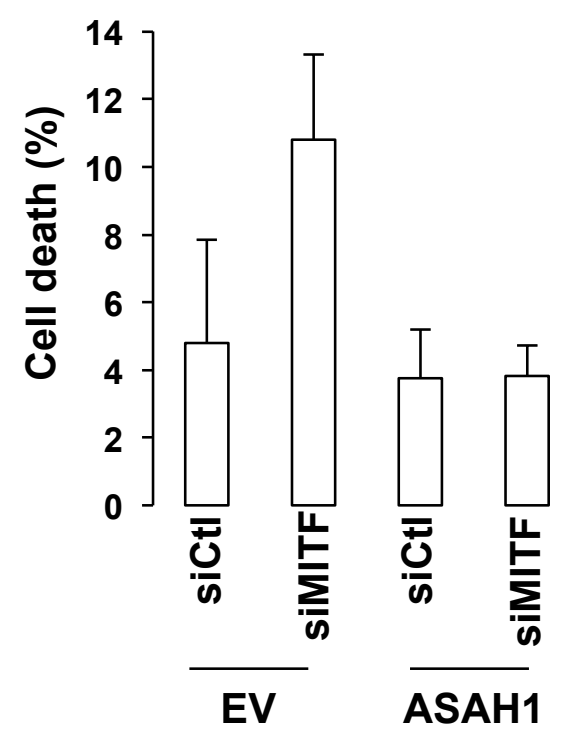


A

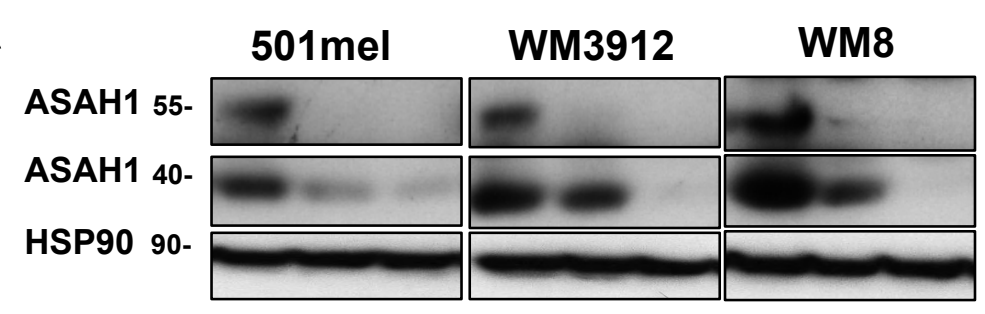

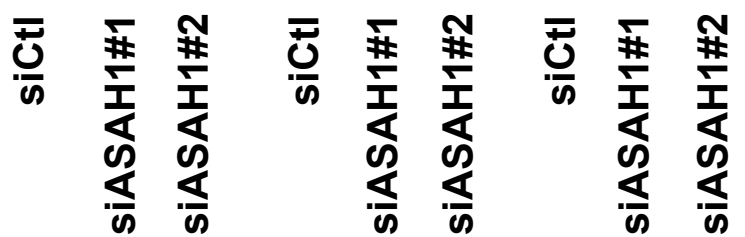
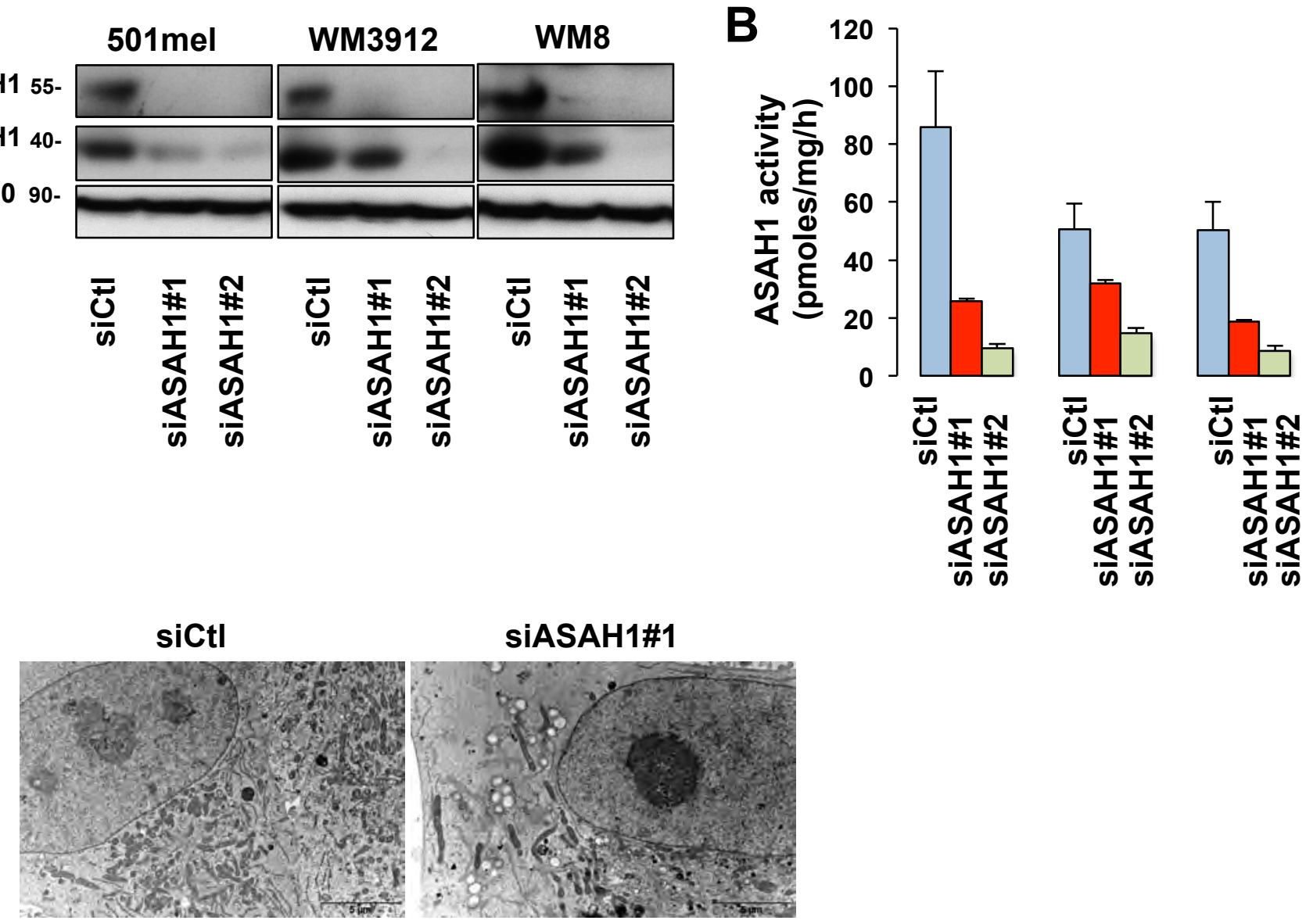

D

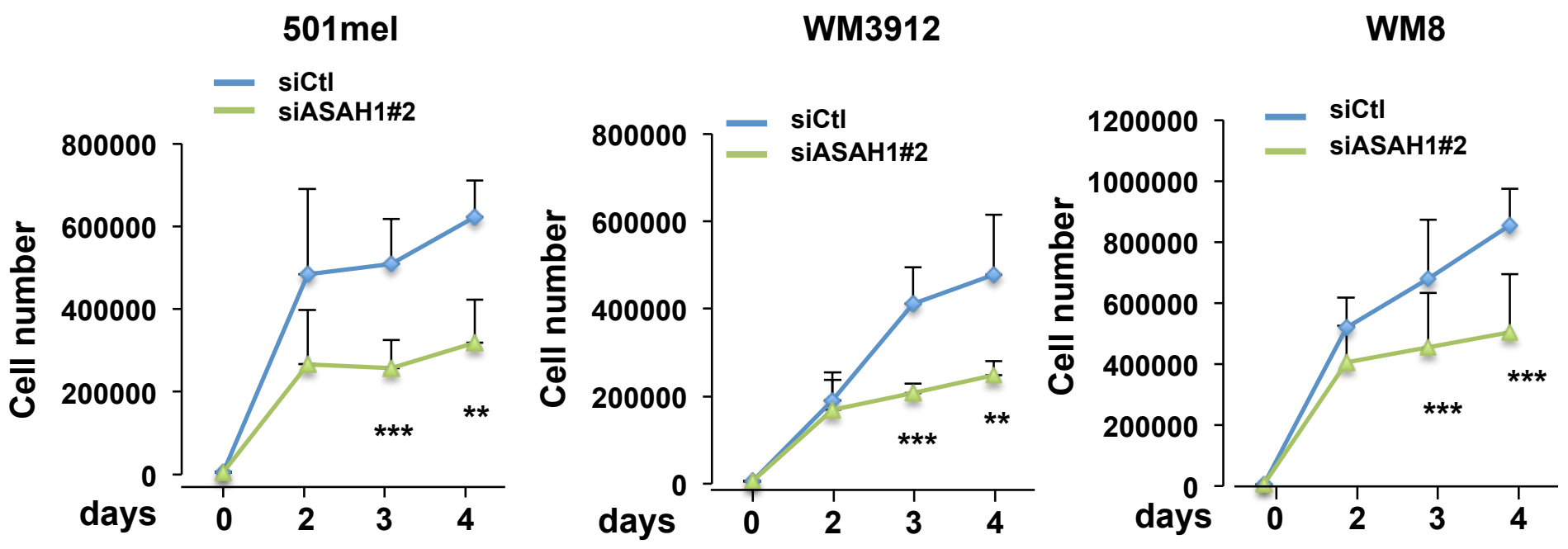


E
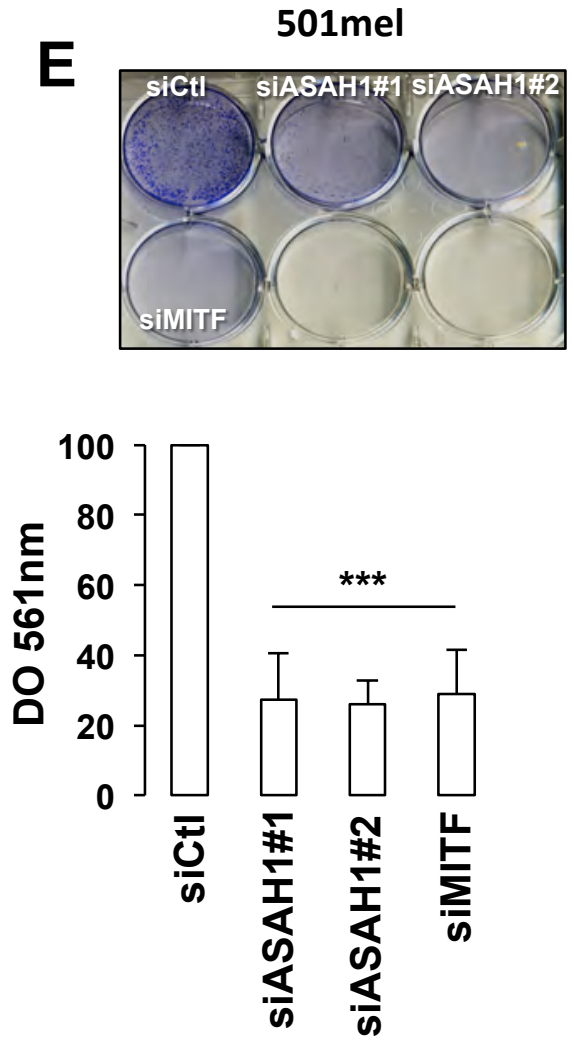

F

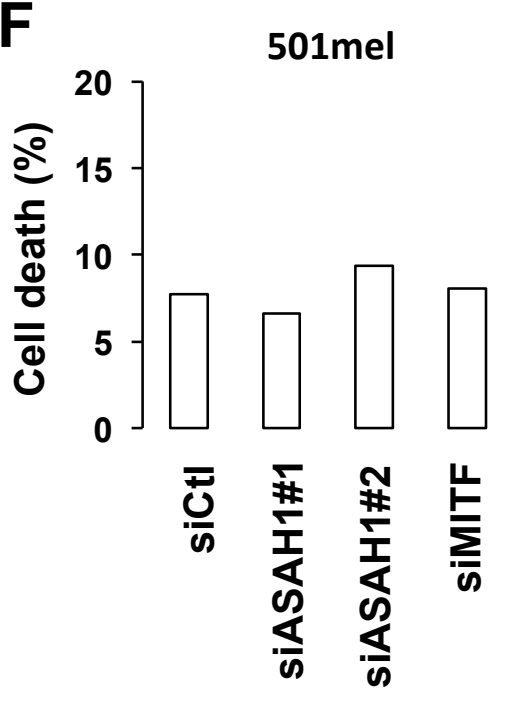

WM3912
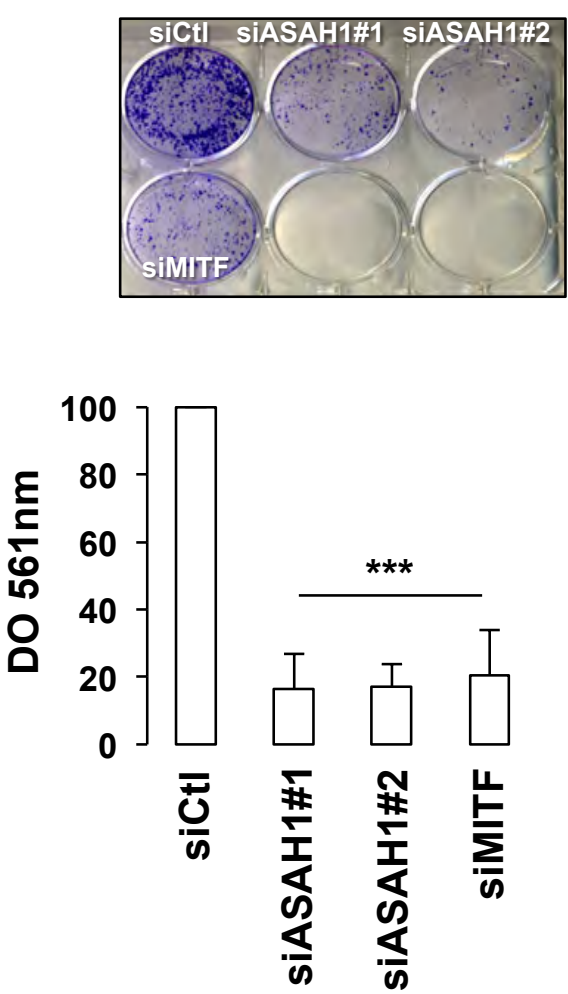

WM3912

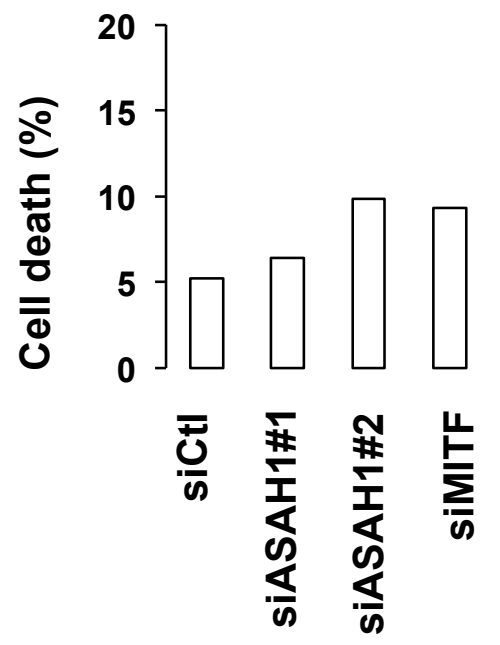

WM8
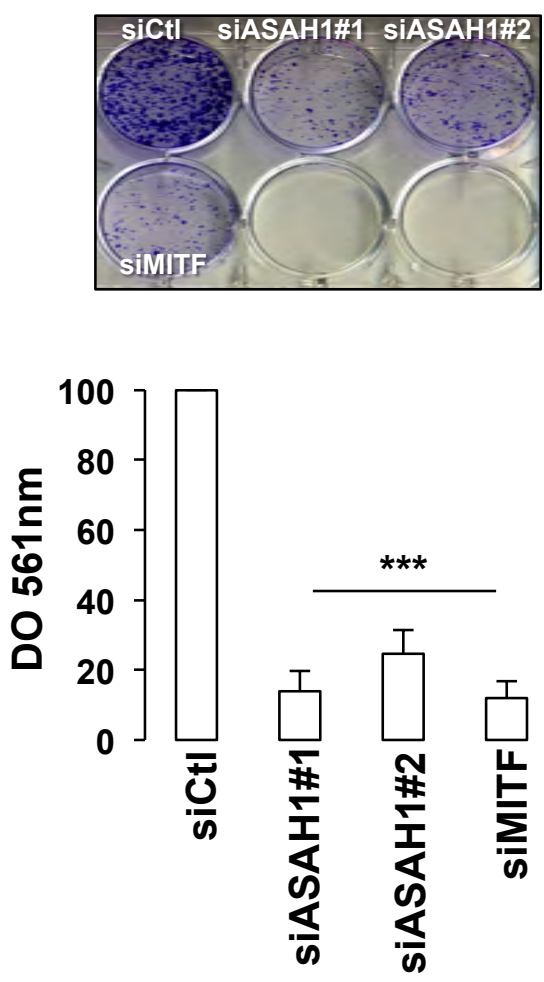

WM8

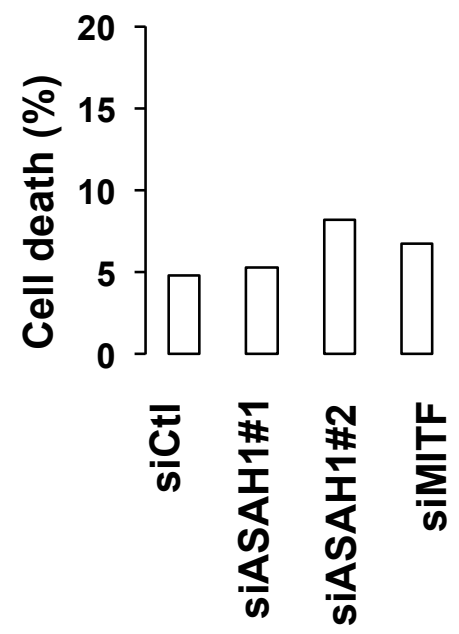




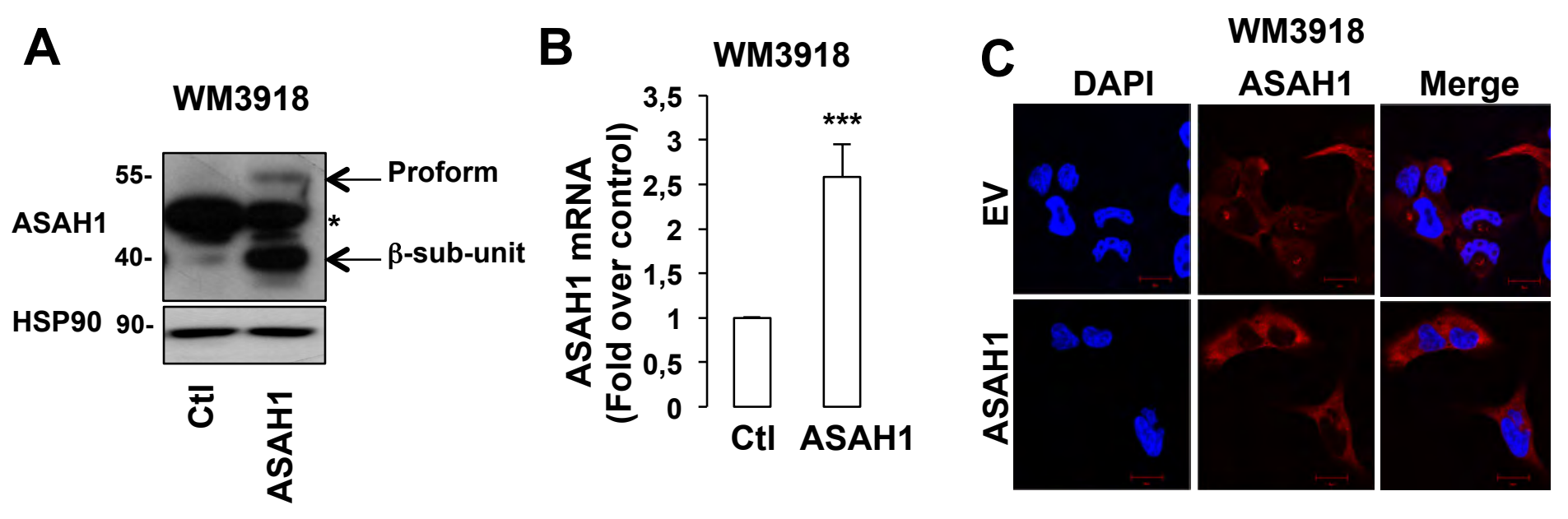

D

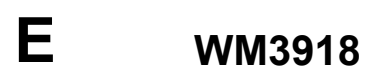

F $\quad$ WM3918
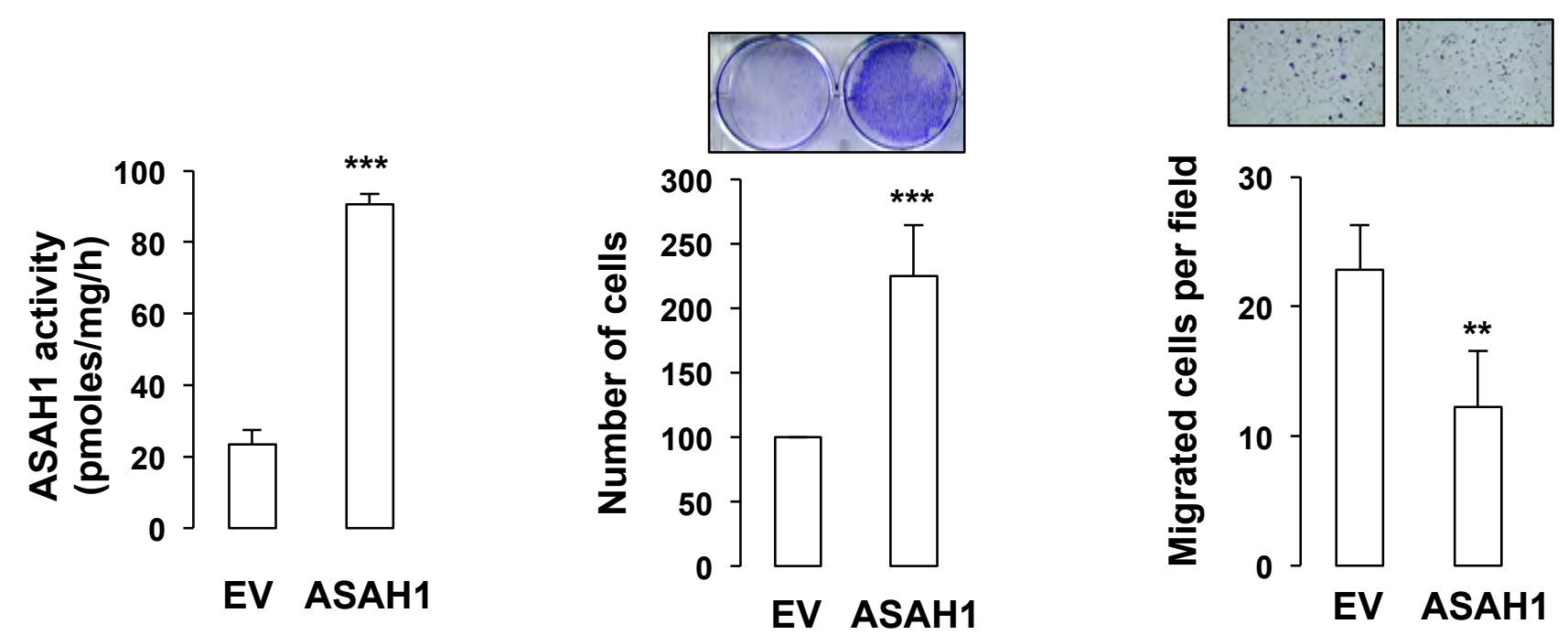

G

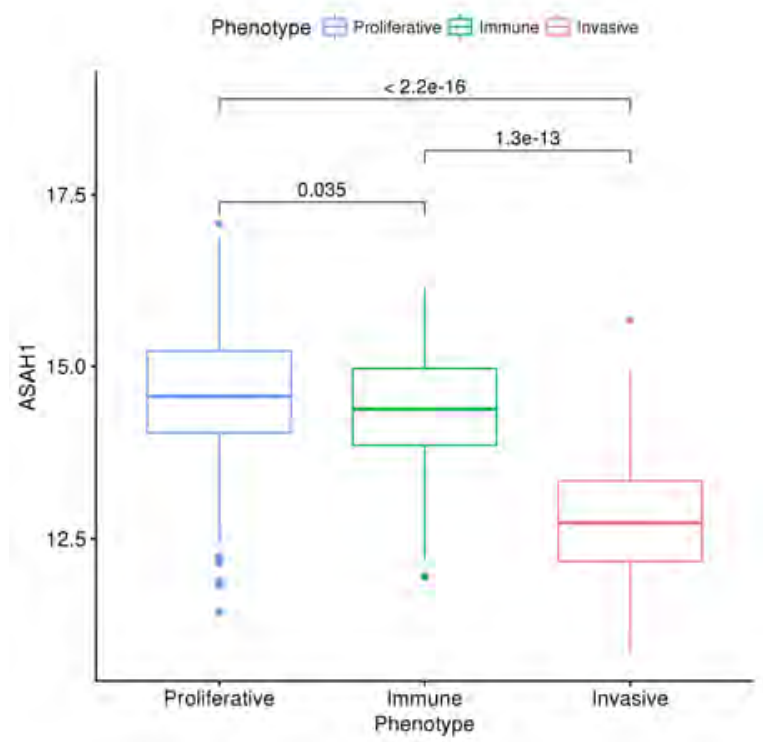


A

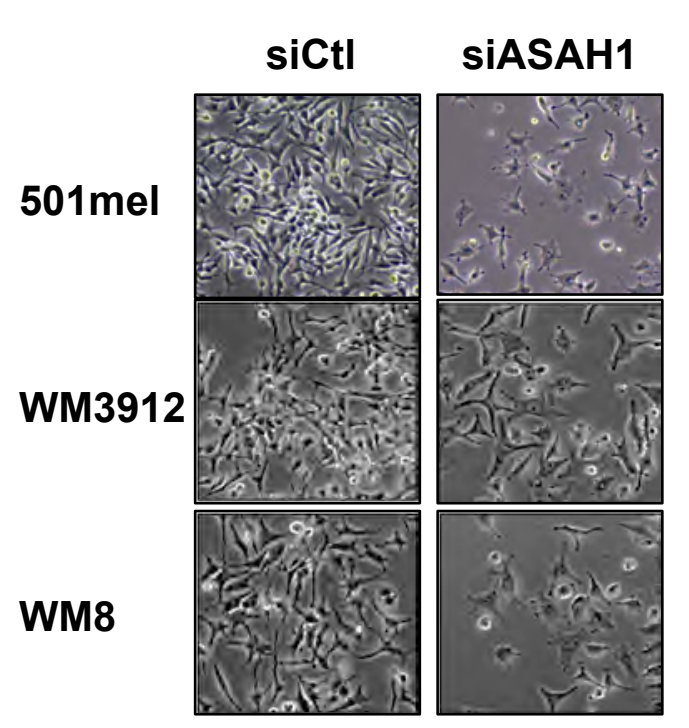

D

$$
\begin{array}{ll}
- & \text { siMITF } \\
& \text { siASAH1 } \\
& \text { siCtl }
\end{array}
$$
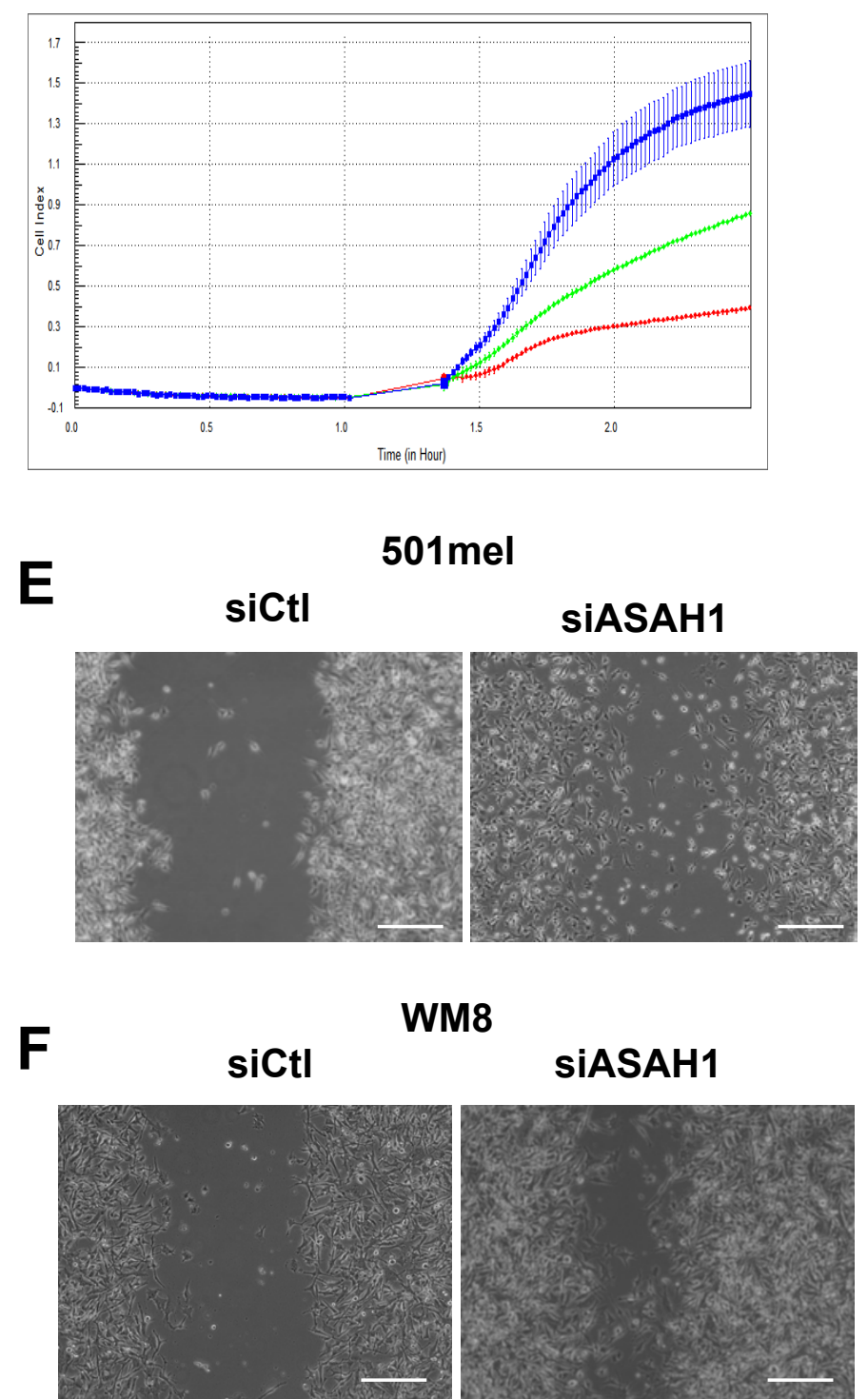

B

WM3918

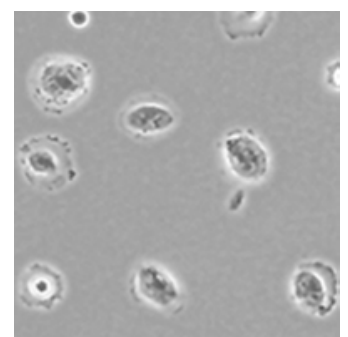

C

$501 \mathrm{mel}$
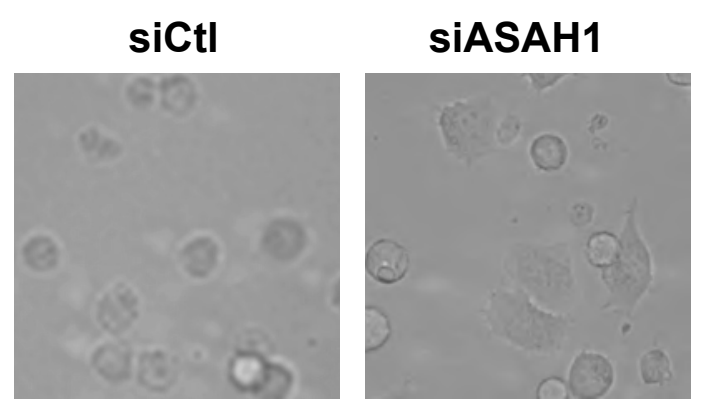

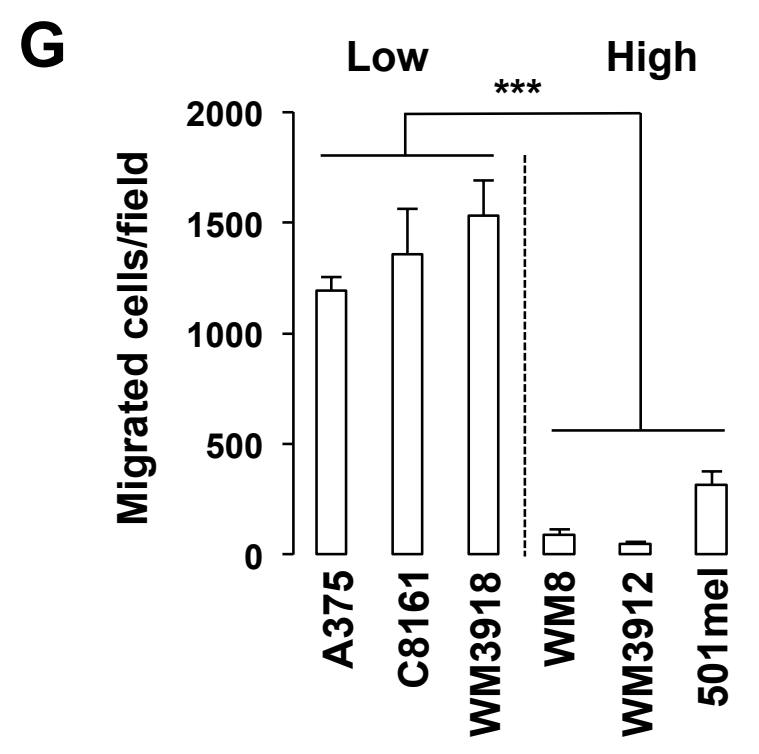

Leclerc_Supplemental figure 5 
A

$501 \mathrm{mel}$

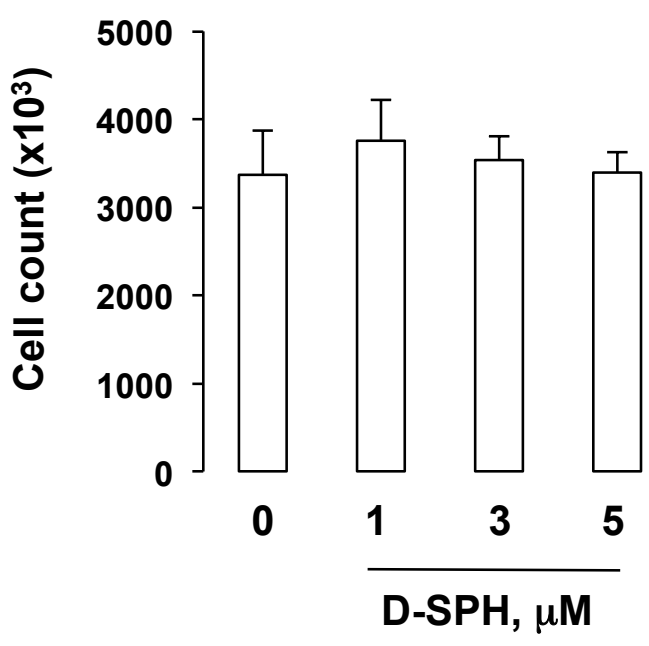

C

WM8

siCtI SIASAH1 SIMITF

Ctl

D-SPH, $3 \mu \mathrm{M}$

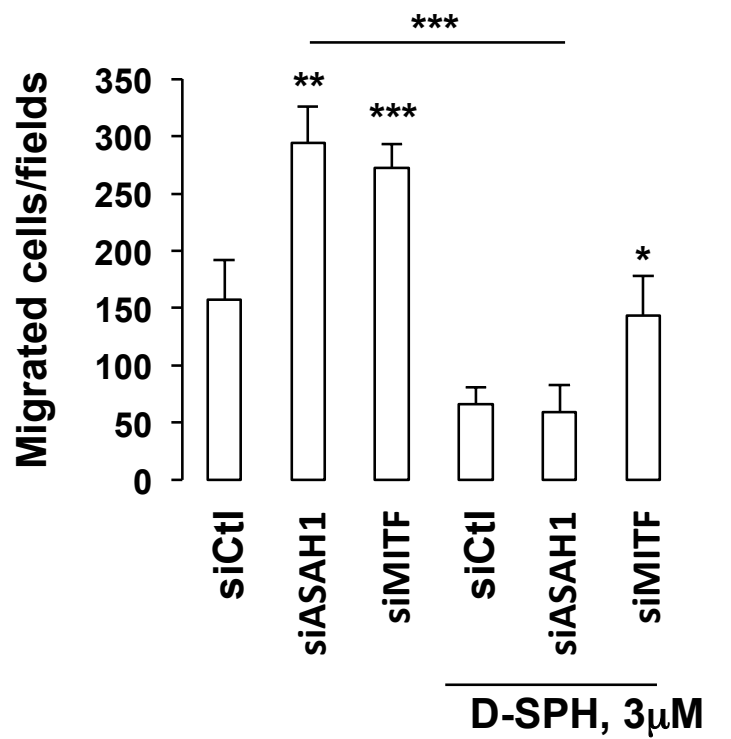

B

$501 \mathrm{mel}$

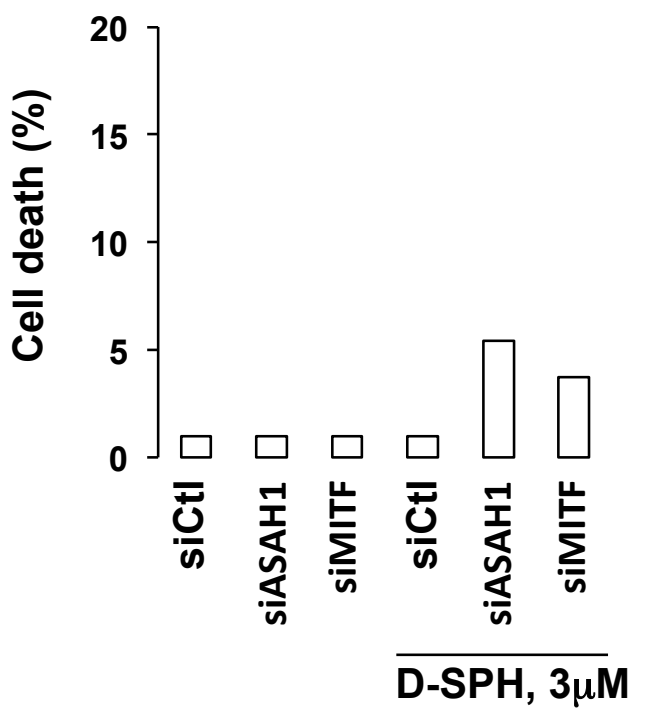

D WM3912 siCtl siASAH1 siMITF

Ctl

D-SPH, $3 \mu \mathrm{M}$

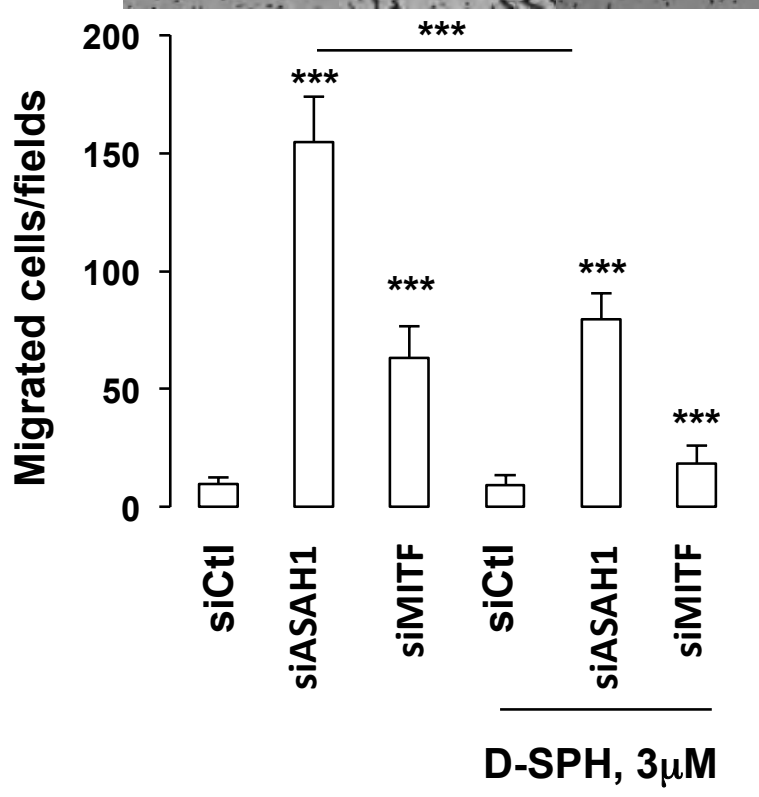


A

B
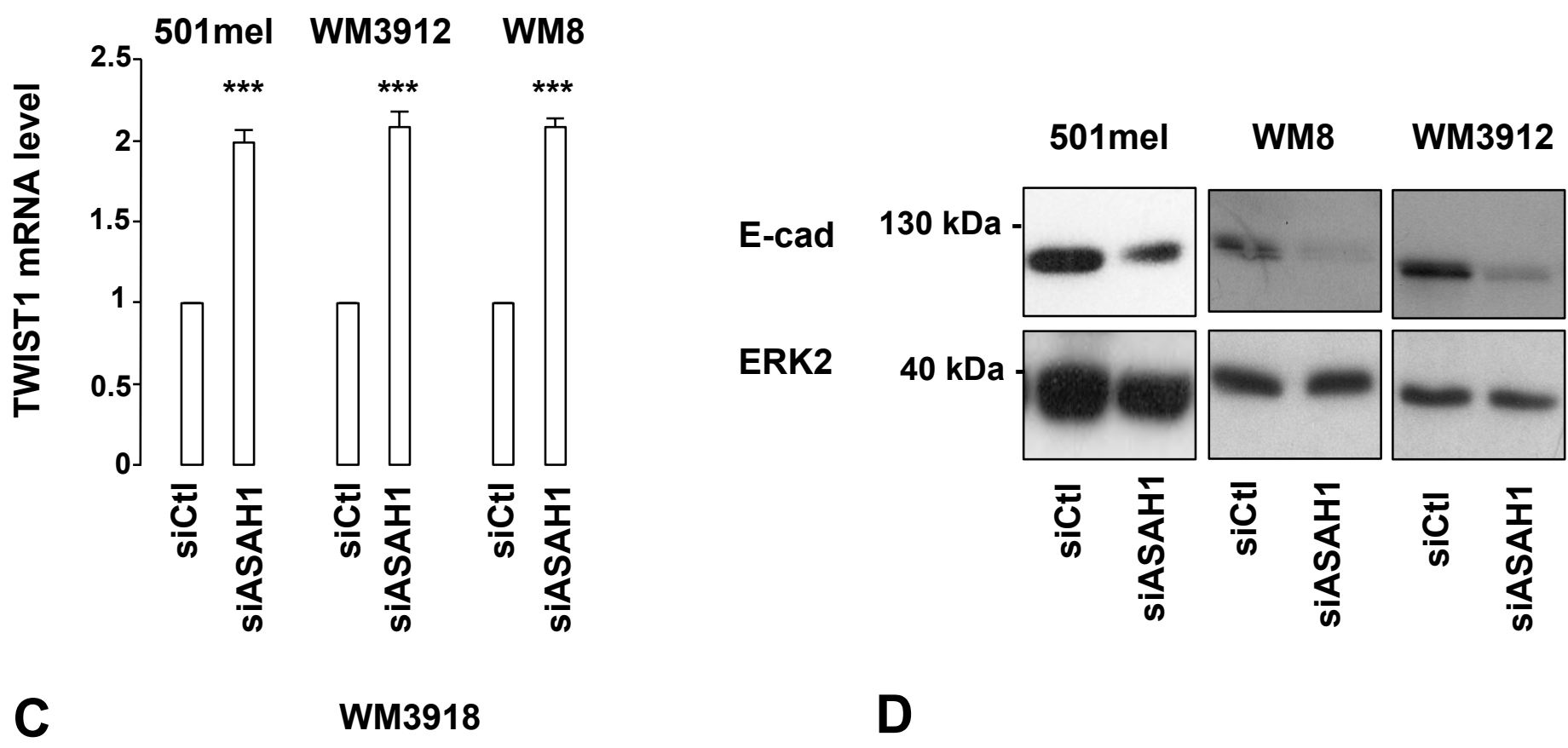

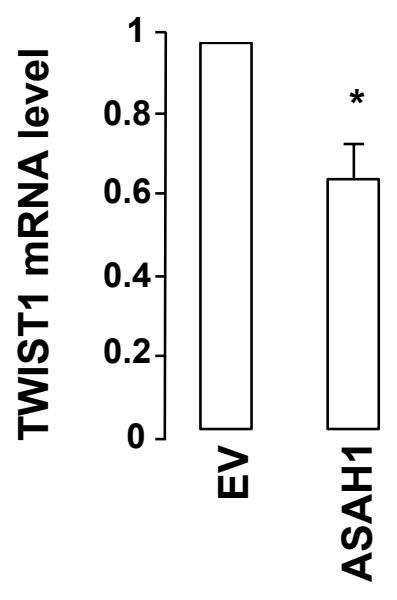

E

$501 \mathrm{mel}$

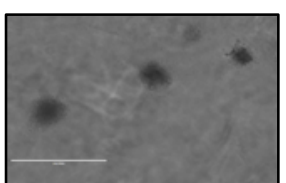

siCtl

SIASAH1

G

501mel
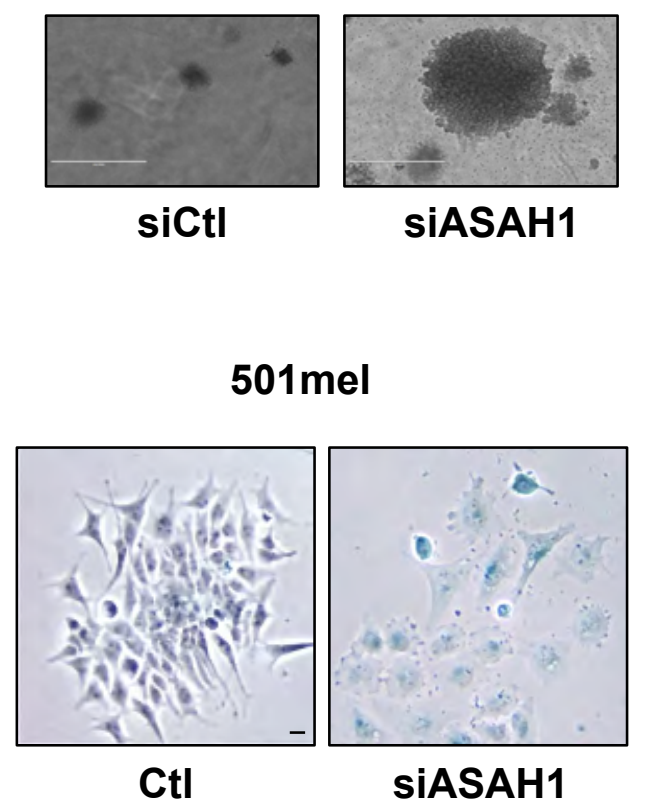

C
WM3918

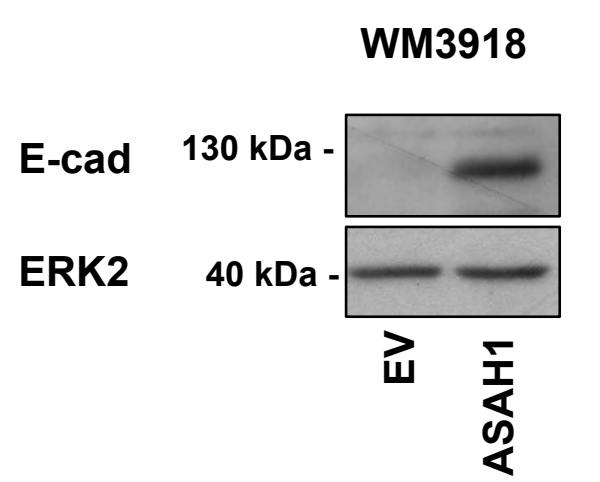

F

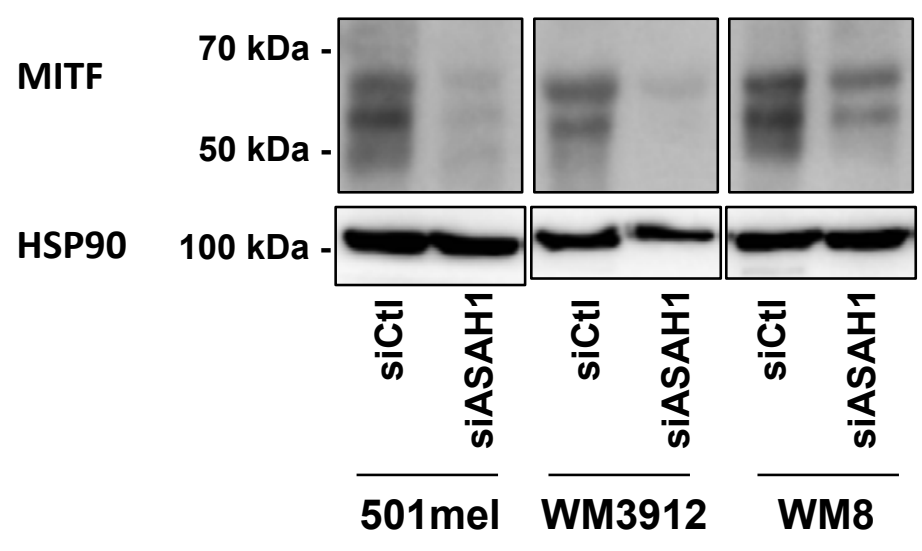

Leclerc_Supplemental figure 7 
A

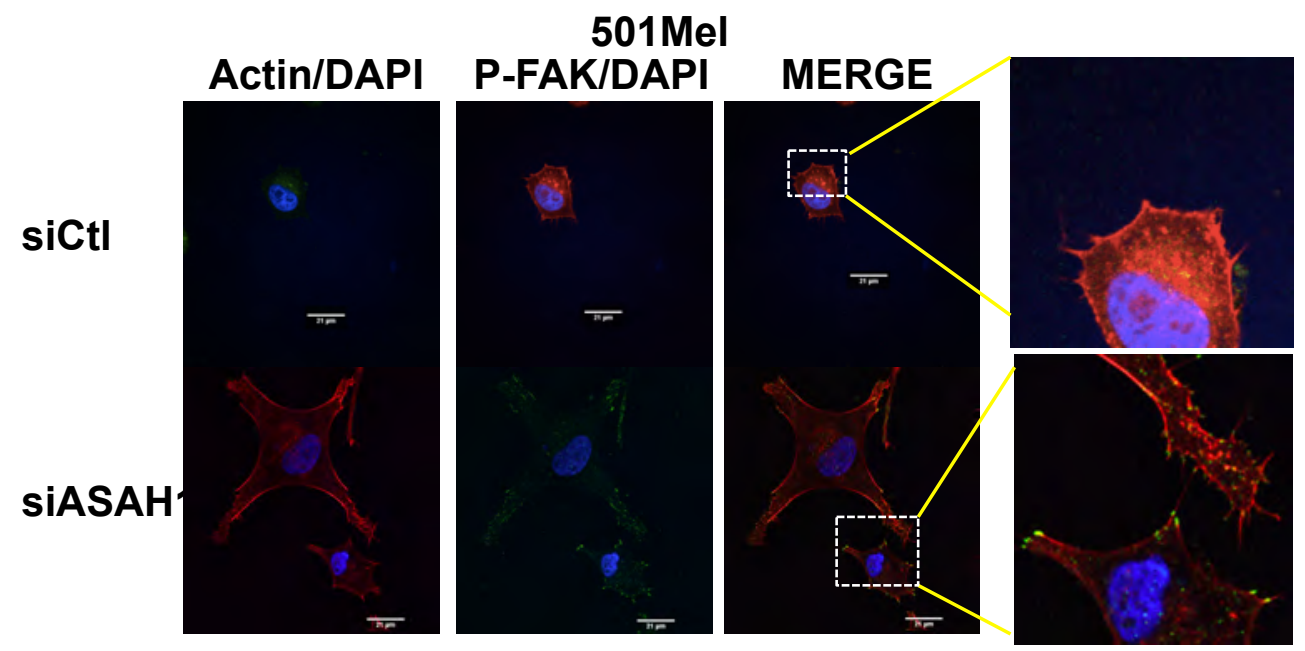

B

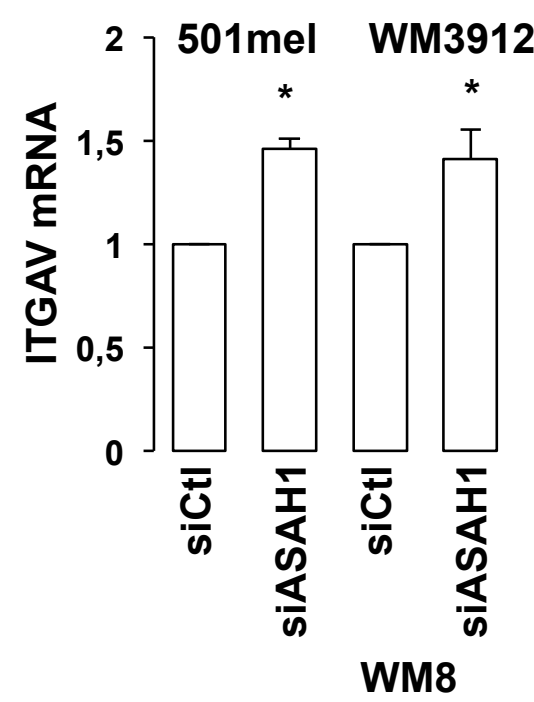

D siCtI SIASAH1 SiMITF

CtI

C

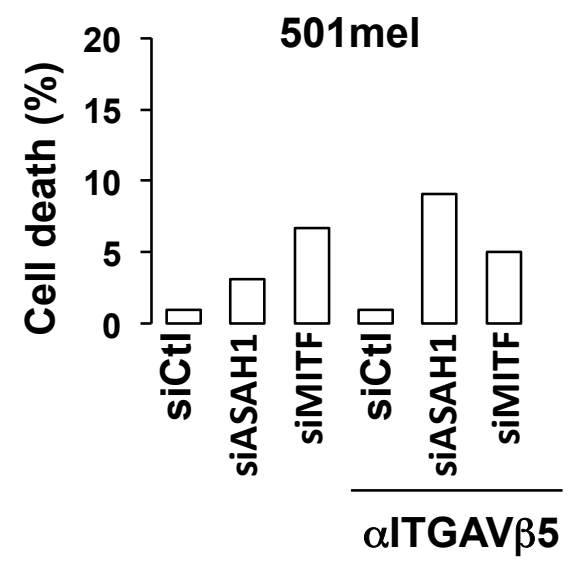

E

WM3912

siCtI SIASAH1 SIMITF

CtI

ITG $\alpha$ V $\beta 5$

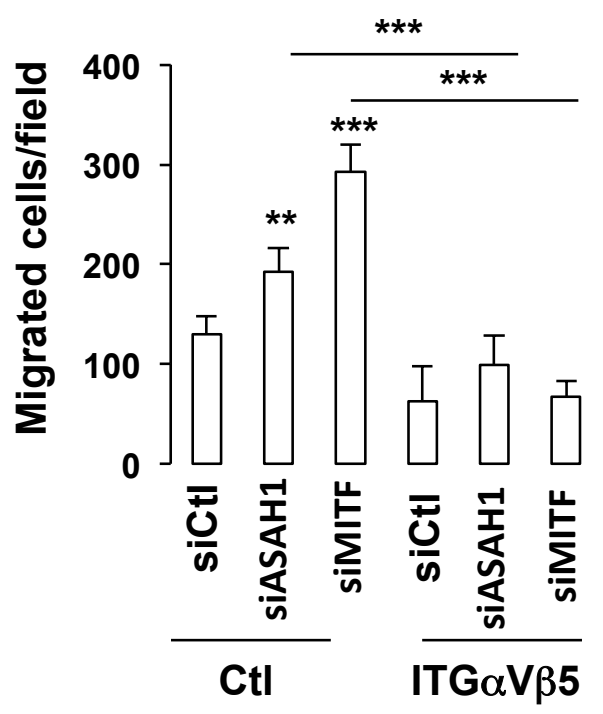

ITG $\alpha \mathbf{V} \beta 5$

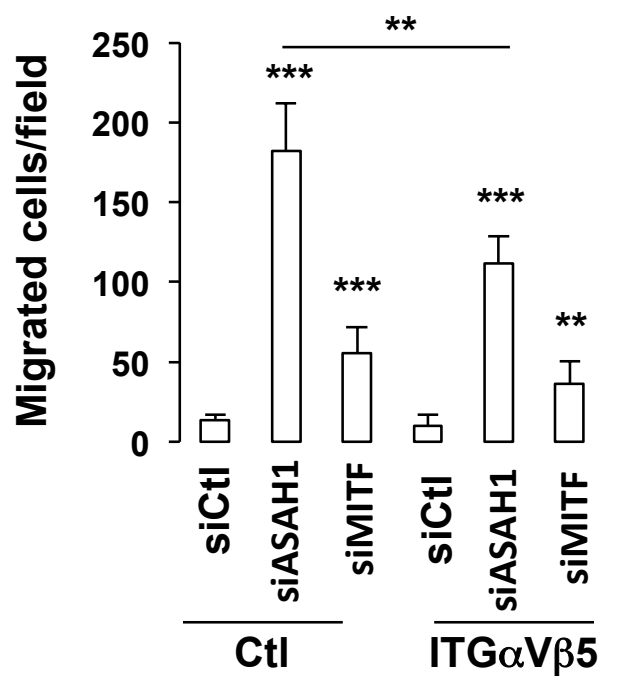

Leclerc_Supplemental figure 8 


\section{Supplementary figures}

Figure S1: ASAH1 displays an elevated expression in melanoma compared to other tumor.

(A) The data from Rambow et al. ${ }^{26}$ were analysed using the Morpheus software (https://software.broadinstitute.org/morpheus/). A relative color scheme based on the minimum and maximum values in each row was used to convert values to color. The relative levels are indicated by varying color intensity (low-blue, high-red).

(B) Correlation between ASAH1 and MITF genes expression in melanoma tumors extracted from dataset GSE19234. Gene expression data were $\log _{2}$ transformed. Pearson's correlation coefficient, used to measure similarity between gene profiles, is shown.

(C) ASAH1 mRNA expression in melanoma cell lines determined by qRT-PCR. Values represent mean $+S D$ of three independent experiments performed in triplicate. ${ }^{* * *} p<0.001$

(D) Immunoblot to ASAH1 with a commercial antibody (HPA005468, Sigma) of total cell extracts isolated from the indicated melanoma cell lines showing the proform (55 $\mathrm{kDa})$ and the $\mathrm{b}(40 \mathrm{kDa})$ active subunits. Detection of ERK2 serves as a loading control. The asterisk indicates a non-specific band.

(E) MITF mRNA expression in melanoma cell lines determined by qRT-PCR. Values represent mean $+S D$ of three independent experiments performed in triplicate. ${ }^{*} p<0.05 ;{ }^{* *} p<0.01 ;{ }^{* * *} p<0.001$.

Figure S2: MITF controls ASAH1 expression 
(A-B) mRNA expression of MITF, two of its target genes (TYROSINASE and MLANA) and ASAH1 in WM3912 and WM8 melanoma cell lines determined by qRTPCR. Values represent mean $+S D$ of three independent experiments performed in triplicate. ${ }^{* * *} p<0.001$.

(C) Cellular lipids were extracted, and sphingolipid concentration was determined by LC/MS in control or MITF-suppressed $501 \mathrm{mel}$ cells. Data represent the levels of sphingosine $(\mathrm{SPH})$ in $\mathrm{ng} / \mathrm{mg}$ proteins.

(D) Immunoblot to MITF and ERK2 (loading control) in 501mel melanoma cells with forced expression of MITF compared to control cells.

(E) ASAH1 mRNA expression in 501mel melanoma cells, transduced with an empty adenoviral vector or a vector encoding MITF (72 hrs), as determined by qRT-PCR. Values represent mean $+S D$ of three independent experiments performed in triplicate. ${ }^{* *} p<0.01$.

(F) Luciferase assay using $501 \mathrm{mel}$ cells transfected with the ASAH1 promoter \{ Lucki, $2009 \# 2845$ \} and with empty pcDNA3 vector or with a vector encoding wild type MITF. Forty-eight hours post-transfection, luciferase activity was assayed and normalized by the $\beta$-galactosidase activity. Results are expressed as fold stimulation of the basal luciferase activity from cells transfected with empty vector. Data are means $+\mathrm{SE}$ of three experiments performed in triplicate. ${ }^{* * *} p<0.001$.

(G) 501mel melanoma cells infected with empty or ASAH1 encoding lentiviruses and transfected with control or MITF siRNA were stained with DAPI. DAPI positive cells were monitored by FACS.

Figure S3: ASAH1 loss inhibits cell proliferation 
(A) Immunoblot to ASAH1 of 501mel (left), WM3912 (middle) and WM8 (right) melanoma cells transfected with a control (siCtl) or two different ASAH1 (siASAH1\#1 and siASAH1\#2) siRNA. The proform (55 kDa) and the $\beta(40 \mathrm{kDa})$ active subunit are shown. The $\alpha(14 \mathrm{kDa})$ active subunit is not recognized by the commercial antibody. Detection of ERK2 serves as a loading control.

(B) ASAH1 activity in the corresponding melanoma cells. Values represent mean + SD of two independent experiments.

(C) TEM images of WM8 melanoma cells transfected with control or ASAH1 siRNA. Scale bars, $5 \mu \mathrm{m}$.

(D) Cell count over time. Values represent mean + SD of three independent experiments. ${ }^{* *} p<0.01 ;{ }^{* * *} p<0.001$.

(E) Colony formation assay of melanoma cells transfected with control, ASAH1 (ASAH1\#1 and ASAH1\#2) or MITF siRNA. Colonies were stained with crystal violet after 14 days (representative micrographs are shown) and destained. The relative absorbance, which reflects the number of colony was measured on a spectrophotometer. Values represent mean + SD of three independent experiments. ${ }^{* * *} p<0.001$.

(F) FACS analysis of DAPI staining in 501mel (left), WM3912 (middle) and WM8 (right) melanoma cells transfected with a control (siCtl), two ASAH1 (siASAH1\#1 and siASAH1\#2) or MITF (siMITF) siRNA. DAPI positive cells represent dying cells. Results are expressed in \%.

\section{Figure S4: ASAH1 controls melanoma cell proliferation}

(A) Immunoblot to ASAH1 of WM3918 melanoma cell lines with forced expression of ASAH1 compared to the parental WM3918 cells. 
(B) Expression of mRNA for ASAH1 measured using qRT-PCR analysis after forced expression of ASAH1 in WM3918 ASAH1 $1^{\text {low }}$ melanoma lines compared to the parental cell lines. Values represent mean + SD of three independent experiments. ${ }^{* * *} p<0.001$.

(C) Confocal microscopy images of ASAH1 in control or expressing ASAH1 WM3918 melanoma cells. Scale bar: $20 \mu \mathrm{m}$

(D) ASAH1 activity after forced expression of ASAH1 in WM918 melanoma cells. Values represent mean $+S D$ of three independent experiments. ${ }^{* * *} p<0.001$.

(E) Colony formation was assessed 10 days later. Values represent mean $+\mathrm{SD}$ of two independent experiments. ${ }^{* * *} p<0.001$. Representative micrographs are shown.

(F) Migration capacity of WM3918 melanoma cell lines with forced expression of ASAH1 compared to the parental cells.

(G) Analysis of ASAH1 association with the three phenotypes (proliferative, invasive, immune) described in Verfaillie et al. \{Verfaillie, 2015 \#2970\} from the TCGA SKCM dataset. P-values were calculated by the Wilcoxon rank sum test.

\section{Figure S5: ASAH1 impacts on melanoma cell adhesion}

(A) Phase contrast microscopy images of melanoma cells upon ASAH1 suppression.

(B) Adhesion assay of ASAH1 $1^{\text {low }}$ melanoma cells infected with control or ASAH1 lentiviruses.

(C) Adhesion assay of ASAH $1^{\text {high }}$ melanoma cells transfected with control or ASAH1 siRNA.

(D) $x$ Celligence impedance assay measuring adhesion of ASAH $1^{\text {high }}$ melanoma cells transfected with control, ASAH1 or MITF siRNA. 
(E-F) Migration ability of 501mel and WM8 melanoma cells was determined using wound healing assays. Pictures from the same area were taken at time 0 and 24 hours after the wound. Representative images are shown. Magnification: 10X and scale bar $=200 \mu \mathrm{m}$.

(G) Migration capacity of representative $A S A H 1^{\text {high }}$ and $A S A H 1^{\text {low }}$ melanoma cell lines with Boyden chamber assay. Values represent mean + SD of three independent experiments. ${ }^{* * *} p<0.001$.

Figure S6: Exogenous sphingosine prevents ASAH1 and MITF-knockdown induced migration

(A) Number of $501 \mathrm{mel}$ melanoma cells in control condition or after treatment with Dsphingosine (D-SPH) $1 \mu \mathrm{M}, 3 \mu \mathrm{M}$ and $5 \mu \mathrm{M}$ for $24 \mathrm{hrs}$.

(B) FACS analysis of DAPI staining in $501 \mathrm{mel}$ melanoma cells transfected with control (siCtl), ASAH1 (siASAH1) or MITF (siMITF) siRNA were left untreated or were incubated with D-sphingosine $(3 \mu \mathrm{M})$ the last 5 hours. Cells positive for DAPI represent dying cells. Results are expressed in \%.

(C-D) Boyden chambers experiments. Cells were transfected for 48 hrs with ASAH1 or MITF siRNA before being exposed to $3 \mu \mathrm{M}$ D-sphingosine (D-SPH) for $5 \mathrm{hrs}$. Values represent mean $+S D$ of three independent experiments. ${ }^{*} p<0.05 ;{ }^{* *} p<0.01$; ${ }^{* * *} p<0.001$

\section{Figure S7: ASAH1 controls mesenchymal features}

(A) TWIST mRNA in 501mel, WM3912 and WM8 melanoma cells transfected with control or ASAH1 siRNA. Values represent mean + SD of three independent experiments. ${ }^{* * *} p<0.001$. 
(B) Immunoblot to E-cadherin and ERK2 (loading control) in 501mel, WM3912 and WM8 melanoma cells transfected with a control or ASAH1 siRNA.

(C) TWIST mRNA in WM3918 with forced expression of ASAH1 compared to the parental cells. Values represent mean $+S D$ of three independent experiments. ${ }^{*} \mathrm{p}<0.05$.

(D) Immunoblot to E cadherin and ERK2 (loading control) in WM3918 melanoma cells with forced expression of ASAH1 compared to parental cells.

(E) Anchorage independent growth of $501 \mathrm{mel}$ melanoma cells transfected with control siRNA or ASAH1 siRNA for $48 \mathrm{hrs.} \mathrm{Colonies} \mathrm{were} \mathrm{observed} \mathrm{after} 10$ days.

(F) Immunoblot to MITF and HSP90 (loading control) in 501mel, WM3912 and WM8 melanoma cells transfected with control siRNA or ASAH1 siRNA. Representative images are shown.

(G) 501mel melanoma cells transfected with control siRNA or ASAH1 siRNA were stained for SA- $\beta-$ Gal activity and visualized under phase-contrast microscopy. Bars, $10 \mu \mathrm{M}$. Representative images are shown.

Figure S8: ASAH1 controls melanoma cells migration through regulation of the integrin/FAK signaling cascade

(A) Confocal microscopy images of localization of phosphorylated FAK (Y397) after forced expression of ASAH1 in 501 mel melanoma cells compared to control cells. Representative images are shown. Scale bar: $21 \mu \mathrm{m}$.

(B) ITGAV mRNA level in ASAH1 depleted cells. Values represent mean + SD of three independent experiments. ${ }^{*} p<0.05$.

(C) FACS analysis of DAPI staining in $501 \mathrm{mel}$ melanoma cells transfected with a control (siCtl), ASAH1 (siASAH1) or MITF (siMITF) siRNA in presence of control lgG 
(Ctl) or ITG $\alpha$ V $\beta 5$ neutralizing antibody (ITG $\alpha \mathrm{V} \beta 5$ ) the last 5 hours. Cells positive for DAPI represent dying cells. Results are expressed in \%.

(D-E) Boyden chamber assays with WM8 and WM3912 melanoma cells transfected with control, ASAH1 or MITF siRNA in presence of control IgG (Ctl) or ITG $\alpha \mathrm{V} \beta 5$ neutralizing antibody. Values represent mean + SD of three independent experiments. Representative images are shown. ${ }^{* *} p<0.01 ;{ }^{* * *} p<0.001$. 